Article

\title{
Kirchhoff-Love Plate Theory: First-Order Analysis, Second-Order Analysis, Plate Buckling Analysis, and Vibration Analysis Using the Finite Difference Method
}

\section{Valentin Fogang}

Civil Engineer, c/o BUNS Sarl, P.O Box 1130, Yaounde, Cameroon; valentin.fogang@bunscameroun.com

ORCID iD https://orcid.org/0000-0003-1256-9862

\begin{abstract}
This paper presents an approach to the Kirchhoff-Love plate theory (KLPT) using the finite difference method (FDM). The KLPT covers the case of small deflections, and shear deformations are not considered. The FDM is an approximate method for solving problems described with differential equations. The FDM does not involve solving differential equations; equations are formulated with values at selected points of the structure. Generally in the case of KLPT, the finite difference approximations are derived based on the fourth-order polynomial hypothesis (FOPH) and second-order polynomial hypothesis (SOPH) for the deflection surface. The FOPH is made for the fourth and third derivative of the deflection surface while the SOPH is made for its second and first derivative; this leads to a 13-point stencil for the governing equation. In addition, the boundary conditions and not the governing equations are applied at the plate edges. In this paper, the FOPH was made for all of the derivatives of the deflection surface; this led to a 25-point stencil for the governing equation. Furthermore, additional nodes were introduced at plate edges and at positions of discontinuity (continuous supports/hinges, incorporated beams, stiffeners, brutal change of stiffness, etc.), the number of additional nodes corresponding to the number of boundary conditions at the node of interest. The introduction of additional nodes allowed us to apply the governing equations at the plate edges and to satisfy the boundary and continuity conditions. First-order analysis, second-order analysis, buckling analysis, and vibration analysis of plates were conducted with this model. Moreover, plates of varying thickness and plates with stiffeners were analyzed. Finally, a direct time integration method (DTIM) was presented. The FDM-based DTIM enabled the analysis of forced vibration of structures, with damping taken into account. In first-order, second-order, buckling, and vibration analyses of rectangular plates, the results obtained in this paper were in good agreement with those of well-established methods, and the accuracy was increased through a grid refinement.
\end{abstract}

Keywords: Kirchhoff-Love plate; finite difference method; additional points; plate of varying thickness; plate with stiffeners; skew edge; plate buckling analysis; vibration analysis; direct time integration method;

\section{Introduction}

This paper describes the application of Fogang's model [1] based on the finite difference method (FDM), used for the Euler-Bernoulli beam, to the Kirchhoff-Love plate. The Kirchhoff-Love plate theory (KLPT) is a twodimensional mathematical model that is used to determine the stresses and deformations in thin plates subjected to 
KIRCHHOFF-LOVE PLATE THEORY USING THE FINITE DIFFERENCE METHOD

forces and moments. This theory is an extension of Euler-Bernoulli beam theory and was developed in 1888 by Love using assumptions proposed by Kirchhoff [2]. KLPT is governed by the Germain-Lagrange plate equation; this equation was first derived by Lagrange in December 1811 in correcting the work of Germain [3] who provided the basis of the theory. The analytical approach to KLPT consists of solving the governing equations that are expressed via means of partial differential equations, and satisfying the boundary and continuity conditions. However, solving the twodimensional partial differential equations may be difficult, especially in the presence of axial forces, an elastic Winkler foundation, plate of varying thickness, etc. Numerical methods permit therefore to overcome solving the differential equations. A considerable volume of literature has been published on numerical methods for Kirchhoff-Love plate analysis. For rectangular plates, Navier [4] in 1820 introduced a simple method for the analysis when a plate is simply supported along all edges; the applied load and the deflection were expressed in terms of Fourier components and double trigonometric series, respectively. Another approach was proposed by Lévy [5] in 1899 for rectangular plates simply supported along two opposite edges; the applied load and the deflection were expressed in terms of Fourier components and simple trigonometric series, respectively. More recently, Kindelan et al. [6] presented a method to obtain optimal finite difference formulas which maximize their frequency range of validity. Both conventional and staggered equispaced stencils for first and second derivatives were considered. Onyia et al. [7] presented the elastic buckling analysis of rectangular thin plates using the single finite Fourier sine integral transform method. Pisacic et al. [8] developed a procedure of calculating deflection of rectangular plate using a finite difference method, programmed in Wolfram Mathematica; the system of equations was built using the mapping function and solved with solve function. Li et al. [9] applied the generalized finite difference method to simulate the bending behavior of functionally graded plates; the governing equations and constrained boundary conditions were derived based on the first-order shear deformation theory and Hamilton's principle. Ferreira et al. [10] proposed a meshless strategy using the Generalized Finite Difference Method upon substitution of the original fourth-order differential equation by a system composed of two second-order partial differential equations; mixed boundary conditions, variable nodal density and curved contours were explored.

In the classical plate analysis using the FDM, the finite difference approximations are derived based on fourth order polynomial hypothesis (FOPH) and second-order polynomial hypothesis (SOPH) in each direction of the deflection surface; the FOPH is made for the fourth and third derivative of the deflection surface while the SOPH is made for the second and first derivative, leading to a 13-point stencil for the governing equation.. In addition, points outside the plate are generally not considered; the boundary conditions are applied at the plate edges and not the governing equations. Consequently, the non-application of the governing equations at the plate edges together with the different polynomial hypotheses for the deflection surface have led to inaccurate results, making the FDM less interesting in comparison to other numerical methods such as the finite element method. In this paper, a model based on FDM was presented. This model consisted of formulating the differential equations with finite differences and introducing additional points at plate edges and at positions of discontinuity (continuous supports/hinges, incorporated beams, stiffeners, brutal change of stiffness's, etc.). The introduction of additional points allowed us to apply the governing equations at the plate edges and to satisfy the boundary and continuity conditions. Furthermore, the finite difference approximations were derived 
using the FOPH for all of the derivatives of the deflection surface; this led to a 25-point stencil for the governing equation. However, for simplification purpose, the SOPH was considered for its second and first derivative at certain positions (plate angles, skew edges). First-order analysis, second-order analysis, plate buckling analysis, and vibration analysis of plates were conducted with this model. Finally, a direct time integration method (DTIM) was presented; the FDM-based DTIM enabled the analysis of forced vibration of structures, the damping being considered.

\section{Materials and methods}

\subsection{First-order analysis of a Kirchhoff plate}

\subsubsection{Governing equations of an isotropic Kirchhoff-Love plate}

The Kirchhoff-Love plate theory (KLPT) [2] is used for thin plates and shear deformations are not considered. The spatial axis convention $(\mathrm{X}, \mathrm{Y}, \mathrm{Z})$ is represented in figure 1 below.

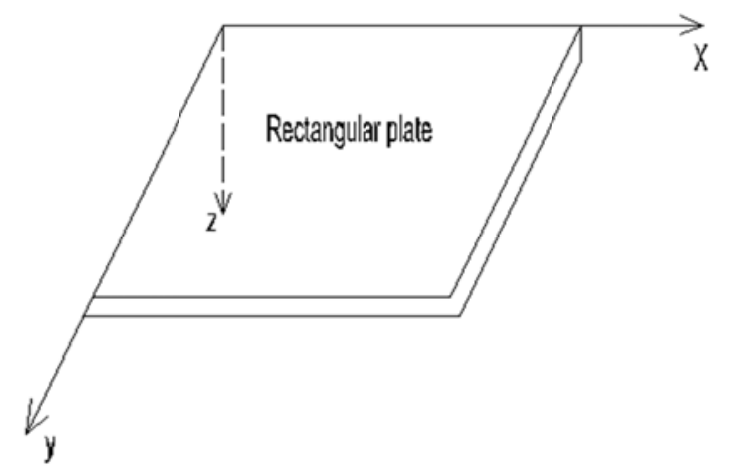

Figure 1 Spatial axis convention X, Y, Z

The equations of the present section are related to the KLPT. The bending moments per unit length $\mathrm{m}_{\mathrm{xx}}$ and $\mathrm{m}_{\mathrm{yy}}$, and the twisting moments per unit length $\mathrm{m}_{\mathrm{xy}}$ are given by

$$
\begin{aligned}
& m_{x x}=-D \times\left(\frac{\partial^{2} w}{\partial x^{2}}+v \frac{\partial^{2} w}{\partial y^{2}}\right), \quad m_{y y}=-D \times\left(\frac{\partial^{2} w}{\partial y^{2}}+v \frac{\partial^{2} w}{\partial x^{2}}\right) \\
& m_{x y}=-D \times(1-v) \times \frac{\partial^{2} w}{\partial x \partial y} \\
& D=\frac{E h^{3}}{12\left(1-v^{2}\right)}
\end{aligned}
$$

The Kirchhoff shear forces per unit length combine shear forces and twisting moments, and can be expressed for an isotropic plate as follows:

$$
V_{x}=-D \times\left(\frac{\partial^{3} w}{\partial x^{3}}+(2-v) \frac{\partial^{3} w}{\partial x \partial y^{2}}\right), \quad V_{y}=-D \times\left(\frac{\partial^{3} w}{\partial y^{3}}+(2-v) \frac{\partial^{3} w}{\partial x^{2} \partial y}\right)
$$


In these equations, $\mathrm{w}(x, y, z)$ is the displacement in $\mathrm{z}$-direction, $\mathrm{D}$ is the flexural rigidity of the plate, $\mathrm{E}$ is the elastic modulus, $\mathrm{d}$ is the plate thickness, and $v$ is the Poisson's ratio. The resulting equilibrium equation is given by

$$
\frac{\partial^{2} m_{x x}}{\partial x^{2}}+2 \frac{\partial^{2} m_{x y}}{\partial x \partial y}+\frac{\partial^{2} m_{y y}}{\partial y^{2}}=-q(x, y),
$$

where q(x,y) denotes the applied transverse load per unit area. Substituting Equations (1a-c) into Equation (3) yields the following governing equation, derived by Lagrange, of the isotropic Kirchhoff plate subjected to a transverse load

$$
\frac{\partial^{4} w}{\partial x^{4}}+2 \frac{\partial^{4} w}{\partial x^{2} \partial y^{2}}+\frac{\partial^{4} w}{\partial y^{4}}=\frac{q(x, y)}{D}
$$

\subsubsection{Finite difference approximations for an isotropic Kirchhoff-Love plate}

\subsubsection{Fundamentals of finite difference approximations}

The governing equation (Equation (4)) has fourth order derivatives; consequently, the deflection surface is approximated around a point of interest $i$ as a fourth degree polynomial in each direction.

Thus, the deflection surface in x-direction i.e. can be described with values of deflections at grid points as follows:

$$
w_{i-2} \times f_{i-2}(x)+w_{i-1} \times f_{i-1}(x)+w_{i} \times f_{i}(x)+w_{i+1} \times f_{i+1}(x)+w_{i+2} \times f_{i+2}(x)
$$

The shape functions $f_{j}(x)(j=i-2 ; i-1 ; i ; i+1 ; i+2)$ can be expressed using the Lagrange interpolating polynomials:

$$
f_{j}(x)=\prod_{\substack{k=i-2 \\ k \neq j}}^{i+2} \frac{x-x_{k}}{x_{j}-x_{k}}
$$

Therefore, a five-point stencil is used to derive finite difference approximations (FDA) to derivatives at grid points. For equidistant grid points with spacing $\boldsymbol{h}$ the derivatives at $\mathbf{i}$ are expressed with values of deflection at points i-2; i-1; i; i+1; $\mathrm{i}+2$.

$$
\begin{aligned}
& \left.\frac{d^{4} w}{d x^{4}}\right|_{i}=\frac{w_{i-2}-4 w_{i-1}+6 w_{i}-4 w_{i+1}+w_{i+2}}{h^{4}} \\
& \left.\frac{d^{3} w}{d x^{3}}\right|_{i}=\frac{-w_{i-2}+2 w_{i-1}-2 w_{i+1}+w_{i+2}}{2 h^{3}} \\
& \left.\frac{d^{2} w}{d x^{2}}\right|_{i}=\frac{-w_{i-2}+16 w_{i-1}-30 w_{i}+16 w_{i+1}-w_{i+2}}{12 h^{2}} \\
& \left.\frac{d w}{d x}\right|_{i}=\frac{w_{i-2}-8 w_{i-1}+8 w_{i+1}-w_{i+2}}{12 h}
\end{aligned}
$$


Applying the Lagrange interpolation polynomials (Equations (5-6)) the first, second, third, and fourth derivatives related to a five-point stencil can be expressed, respectively, as follows

$$
\left[\begin{array}{c}
w_{i-2}^{\prime} \\
w_{i-1}^{\prime} \\
w_{i}^{\prime} \\
w_{i+1}^{\prime} \\
w_{i+2}^{\prime}
\end{array}\right]=\frac{1}{12 h}\left[\begin{array}{ccccc}
-25 & 48 & -36 & 16 & -3 \\
-3 & -10 & 18 & -6 & 1 \\
1 & -8 & 0 & 8 & -1 \\
-1 & 6 & -18 & 10 & 3 \\
3 & -16 & 36 & -48 & 25
\end{array}\right] \times\left[\begin{array}{c}
w_{i-2} \\
w_{i-1} \\
w_{i} \\
w_{i+1} \\
w_{i+2}
\end{array}\right]
$$$$
\left[\begin{array}{c}
w^{(2)}{ }_{i-2}^{(2)} \\
w^{(2)}{ }_{i-1}^{(2)} \\
w^{(2)}{ }_{i}^{(2)} \\
w^{(2)}{ }_{i+1}^{(2)} \\
w^{i+2}
\end{array}\right]=\frac{1}{12 h^{2}}\left[\begin{array}{ccccc}
35 & -104 & 114 & -56 & 11 \\
11 & -20 & 6 & 4 & -1 \\
-1 & 16 & -30 & 16 & -1 \\
-1 & 4 & 6 & -20 & 11 \\
11 & -56 & 114 & -104 & 35
\end{array}\right] \times\left[\begin{array}{c}
w_{i-2} \\
w_{i-1} \\
w_{i} \\
w_{i+1} \\
w_{i+2}
\end{array}\right]
$$

$$
\left[\begin{array}{c}
w^{(3)}{ }_{i-2} \\
w^{(3)}{ }_{i-1}^{(3)} \\
w^{(3)}{ }_{i}^{(3)}{ }^{(3+1} \\
w^{(3)}{ }_{i+2}
\end{array}\right]=\frac{1}{2 h^{3}}\left[\begin{array}{ccccc}
-5 & 18 & -24 & 14 & -3 \\
-3 & 10 & -12 & 6 & -1 \\
-1 & 2 & 0 & -2 & 1 \\
1 & -6 & 12 & -10 & 3 \\
3 & -14 & 24 & -18 & 5
\end{array}\right] \times\left[\begin{array}{c}
w_{i-2} \\
w_{i-1} \\
w_{i} \\
w_{i+1} \\
w_{i+2}
\end{array}\right]
$$

$$
\left.\frac{d^{4} w}{d x^{4}}\right|_{i-2}=\left.\frac{d^{4} w}{d x^{4}}\right|_{i-1}=\left.\frac{d^{4} w}{d x^{4}}\right|_{i}=\left.\frac{d^{4} w}{d x^{4}}\right|_{i+1}=\left.\frac{d^{4} w}{d x^{4}}\right|_{i+2}=\frac{w_{i-2}-4 w_{i-1}+6 w_{i}-4 w_{i+1}+w_{i+2}}{h^{4}}
$$

However, FDAs of the first and second derivatives are sometimes expressed using second order polynomial hypothesis in each direction of the deflection surface as follows

$$
\left.\frac{\partial^{2} w}{\partial x^{2}}\right|_{i-1}=\left.\frac{\partial^{2} w}{\partial x^{2}}\right|_{i}=\left.\frac{\partial^{2} w}{\partial x^{2}}\right|_{i+1}=\frac{w_{i-1}-2 w_{i}+w_{i+1}}{h^{2}},\left.\quad \frac{\partial w}{\partial x}\right|_{i}=\frac{-w_{i-1}+w_{i+1}}{2 h}
$$

Given the spacings $\Delta \mathbf{x}=\mathbf{h}$ and $\Delta \mathbf{y}=\lambda \mathbf{h}$, the derivative $\partial^{2} \mathrm{w} / \partial \mathrm{x} \partial \mathrm{y}$ is then obtained using Equation (7j) as follows

$$
\left.\frac{\partial^{2} w}{\partial x \partial y}\right|_{i}=\frac{1}{4 \lambda h^{2}}\left[\begin{array}{ccc}
1 & 0 & -1 \\
0 & {[0]} & 0 \\
-1 & 0 & 1
\end{array}\right] \times[w]
$$




\subsubsection{Finite difference approximations at an interior node}

Figure 2 shows a plate having equidistant nodes with spacings $\Delta \mathbf{x}$ and $\Delta \mathbf{y}$ in $\mathrm{x}$ - and y-direction, respectively. The node of interest $\mathbf{k}$ and the surrounding nodes are represented, whereby n, s, e, and w stand for the directions north, south, east, and west, respectively, according to the directions in the stencil. The node k may even be at the boundary of the plate.



Figure 2 Point of interest $\mathbf{k}$ and its surrounding points

Assuming a fourth order polynomial hypothesis or a second order polynomial hypothesis for the deflection surface in each direction, this leads to a five-point stencil or a three-point stencil, respectively, for the FDA of the second partial derivative of the deflection surface. The governing equation (Equation (4)) can then be described using a 25-point stencil or a 13-point stencil.

Governing equation, moments, and Kirchhoff shear forces using a 25-point stencil

We set $\Delta \mathbf{x}=\mathbf{h}$ and $\Delta \mathbf{y}=\lambda \mathbf{h}$. Applying Equation (7a) yields the FDA of the fourth derivatives in Equation (4) as follows

$$
\begin{aligned}
& \left.\frac{\partial^{4} w}{\partial x^{4}}\right|_{k}=\frac{w_{w w}-4 w_{w}+6 w_{k}-4 w_{e}+w_{e e}}{h^{4}} \\
& \left.\frac{\partial^{4} w}{\partial y^{4}}\right|_{k}=\frac{w_{s s}-4 w_{s}+6 w_{k}-4 w_{n}+w_{n n}}{(\lambda h)^{4}}
\end{aligned}
$$


The twisting term $\partial^{4} \mathrm{w} / \partial \mathrm{x}^{2} \partial \mathrm{y}^{2}$ defined using Equation (7c) is expressed by means of the following 25-point stencil

$$
\left.\frac{\partial^{4} w}{\partial x^{2} \partial y^{2}}\right|_{k}=\frac{1}{144 \lambda^{2} h^{4}}\left[\begin{array}{ccccc}
1 & -16 & 30 & -16 & 1 \\
-16 & 256 & -480 & 256 & -16 \\
30 & -480 & {[900]} & -480 & 30 \\
-16 & 256 & -480 & 256 & -16 \\
1 & -16 & 30 & -16 & 1
\end{array}\right] \times[w]
$$

We set

$$
\mathrm{W}(\mathrm{x}, \mathrm{y})=\mathrm{D} \times \mathrm{w}(\mathrm{x}, \mathrm{y})
$$

In the stencils, the point of interest is in brackets. Substituting Equations (8a-d) into Equation (4) yields the stencil notation of the governing equation (Equation (4)) of the plate as follows

$\frac{1}{h^{4}}\left[\begin{array}{ccccc}\frac{1}{72 \lambda^{2}} & \frac{-2}{9 \lambda^{2}} & \frac{1}{\lambda^{4}}+\frac{5}{12 \lambda^{2}} & \frac{-2}{9 \lambda^{2}} & \frac{1}{72 \lambda^{2}} \\ \frac{-2}{9 \lambda^{2}} & \frac{32}{9 \lambda^{2}} & \frac{-4}{\lambda^{4}}-\frac{20}{3 \lambda^{2}} & \frac{32}{9 \lambda^{2}} & \frac{-2}{9 \lambda^{2}} \\ 1+\frac{5}{12 \lambda^{2}} & -4-\frac{20}{3 \lambda^{2}} & {\left[6+\frac{6}{\lambda^{4}}+\frac{25}{2 \lambda^{2}}\right]} & -4-\frac{20}{3 \lambda^{2}} & 1+\frac{5}{12 \lambda^{2}} \\ \frac{-2}{9 \lambda^{2}} & \frac{32}{9 \lambda^{2}} & \frac{-4}{\lambda^{4}}-\frac{20}{3 \lambda^{2}} & \frac{32}{9 \lambda^{2}} & \frac{-2}{9 \lambda^{2}} \\ \frac{1}{72 \lambda^{2}} & \frac{-2}{9 \lambda^{2}} & \frac{1}{\lambda^{4}}+\frac{5}{12 \lambda^{2}} & \frac{-2}{9 \lambda^{2}} & \frac{1}{72 \lambda^{2}}\end{array}\right] \times[W]=q_{i}$

In case of a uniform grid $\Delta \mathbf{x}=\Delta \mathbf{y}=\mathbf{h}$, Equation (8e) becomes

$$
\left[\begin{array}{ccccc}
\frac{1}{72} & \frac{-2}{9} & \frac{17}{12} & \frac{-2}{9} & \frac{1}{72} \\
\frac{-2}{9} & \frac{32}{9} & -\frac{32}{3} & \frac{32}{9} & \frac{-2}{9} \\
\frac{17}{12} & -\frac{32}{3} & {[24.5]} & -\frac{32}{3} & \frac{17}{12} \\
\frac{-2}{9} & \frac{32}{9} & -\frac{32}{3} & \frac{32}{9} & \frac{-2}{9} \\
\frac{1}{72} & \frac{-2}{9} & \frac{17}{12} & \frac{-2}{9} & \frac{1}{72}
\end{array}\right] \times[W]=q_{i}
$$


For simplification purpose, Poisson's ratio $v=0$. Applying Equations (7b-d) and (8d) in Equations (1a-c) and (2a-b) yields the bending/twisting moments per unit length and the Kirchhoff shear forces per unit length, respectively, as follows:

$m_{x x, k}=\frac{1}{12 h^{2}}\left[\begin{array}{lllll}1 & -16 & {[30]} & -16 & 1\end{array}\right] \times[W]$

$m_{y y, k}=\frac{1}{12 \lambda^{2} h^{2}}\left[\begin{array}{c}1 \\ -16 \\ {[30]} \\ -16 \\ 1\end{array}\right] \times[W] \quad m_{x y, k}=\frac{1}{144 \lambda h^{2}}\left[\begin{array}{ccccc}-1 & 8 & 0 & -8 & 1 \\ 8 & -64 & 0 & 64 & -8 \\ 0 & 0 & {[0]} & 0 & 0 \\ -8 & 64 & 0 & -64 & 8 \\ 1 & -8 & 0 & 8 & -1\end{array}\right] \times[W]$

$V_{x, k}=\frac{1}{2 h^{3}}\left[\begin{array}{ccccc}\frac{1}{36 \lambda^{2}} & \frac{-2}{9 \lambda^{2}} & 0 & \frac{2}{9 \lambda^{2}} & \frac{-1}{36 \lambda^{2}} \\ \frac{-4}{9 \lambda^{2}} & \frac{32}{9 \lambda^{2}} & 0 & \frac{-32}{9 \lambda^{2}} & \frac{4}{9 \lambda^{2}} \\ 1+\frac{5}{6 \lambda^{2}} & -2-\frac{20}{3 \lambda^{2}} & {[0]} & 2+\frac{20}{3 \lambda^{2}} & -1-\frac{5}{6 \lambda^{2}} \\ \frac{-4}{9 \lambda^{2}} & \frac{32}{9 \lambda^{2}} & 0 & \frac{-32}{9 \lambda^{2}} & \frac{4}{9 \lambda^{2}} \\ \frac{1}{36 \lambda^{2}} & \frac{-2}{9 \lambda^{2}} & 0 & \frac{2}{9 \lambda^{2}} & \frac{-1}{36 \lambda^{2}}\end{array}\right] \times[W]$

$V_{y, k}=\frac{1}{2 h^{3}}\left[\begin{array}{ccccc}\frac{1}{36 \lambda} & \frac{-4}{9 \lambda} & \frac{1}{\lambda^{3}}+\frac{5}{6 \lambda} & \frac{-4}{9 \lambda} & \frac{1}{36 \lambda} \\ \frac{-2}{9 \lambda} & \frac{32}{9 \lambda} & -\frac{2}{\lambda^{3}}-\frac{20}{3 \lambda} & \frac{32}{9 \lambda} & \frac{-2}{9 \lambda} \\ 0 & 0 & {[0]} & 0 & 0 \\ \frac{2}{9 \lambda} & \frac{-32}{9 \lambda} & \frac{2}{\lambda^{3}}+\frac{20}{3 \lambda} & \frac{-32}{9 \lambda} & \frac{2}{9 \lambda} \\ \frac{-1}{36 \lambda} & \frac{4}{9 \lambda} & -\frac{1}{\lambda^{3}}-\frac{5}{6 \lambda} & \frac{4}{9 \lambda} & \frac{-1}{36 \lambda}\end{array}\right] \times[W]$ 
Governing equation, moments, and Kirchhoff shear forces using a 13-point stencil

Equations (8a-b) also applied for the bending terms $\partial^{4} w / \partial x^{4}$ and $\partial^{4} w / \partial y^{4}$. The twisting term $\partial^{4} w / \partial x^{2} \partial y^{2}$ defined using Equation (7i) is expressed by means of the following 9-point stencil

$$
\left.\frac{\partial^{4} w}{\partial x^{2} \partial y^{2}}\right|_{k}=\frac{1}{\lambda^{2} h^{4}}\left[\begin{array}{ccc}
1 & -2 & 1 \\
-2 & {[4]} & -2 \\
1 & -2 & 1
\end{array}\right] \times[w]
$$

Substituting Equations (8a-b), (8d), and (9f) into Equation (4) yields the following widely known 13-point stencil notation of the governing equation (Equation (4)) of the plate

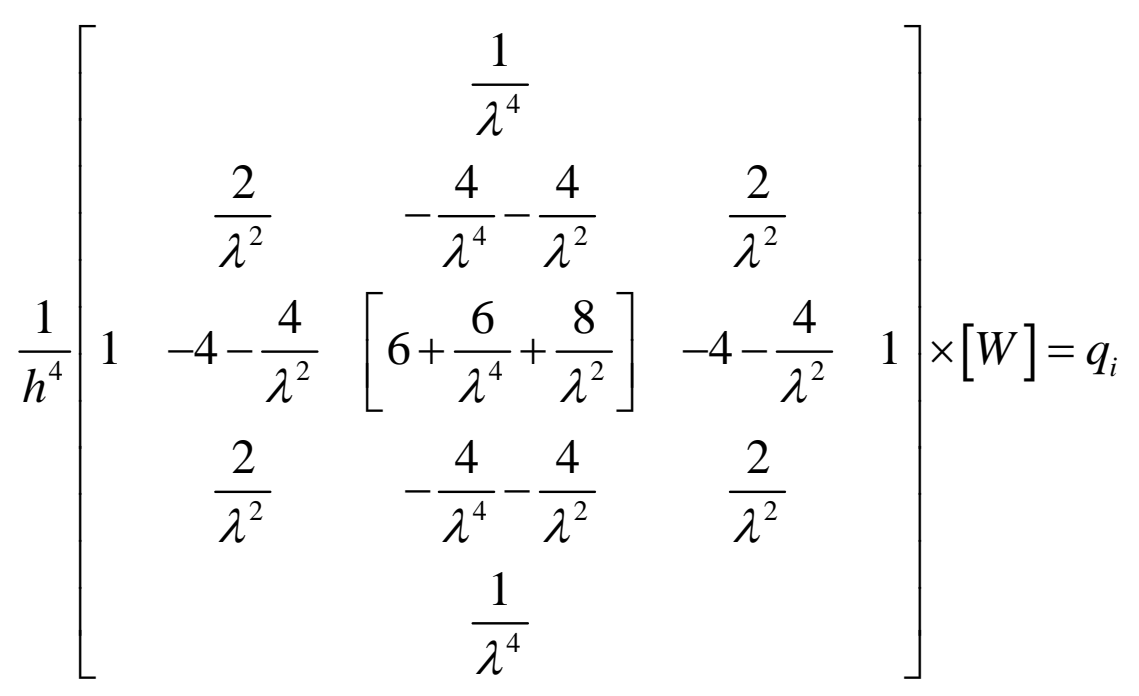

In case of a uniform grid $\Delta \mathbf{x}=\Delta \mathbf{y}=\mathbf{h}$, Equation (9g) becomes

$$
\frac{1}{h^{4}}\left[\begin{array}{ccccc} 
& & 1 & & \\
& 2 & -8 & 2 & \\
1 & -8 & {[20]} & -8 & 1 \\
& 2 & -8 & 2 & \\
& & 1 &
\end{array}\right] \times[W]=q_{i}
$$

Setting Poisson's ratio $v=0$, the application of Equations (7i-j) in Equations (1a-c) and (2a-b) yields the bending/twisting moments per unit length and the Kirchhoff shear forces per unit length, respectively, as follows:

$$
\begin{aligned}
& m_{x x, k}=-\frac{1}{h^{2}}\left[\begin{array}{lll}
1 & {[-2]} & 1
\end{array}\right] \times[W] \\
& m_{y y, k}=-\frac{1}{\lambda^{2} h^{2}}\left[\begin{array}{c}
1 \\
{[-2]} \\
1
\end{array}\right] \times[W], \quad m_{x y, k}=-\frac{1}{4 \lambda h^{2}}\left[\begin{array}{ccc}
1 & 0 & -1 \\
0 & {[0]} & 0 \\
-1 & 0 & 1
\end{array}\right] \times[W]
\end{aligned}
$$


KIRCHHOFF-LOVE PLATE THEORY USING THE FINITE DIFFERENCE METHOD

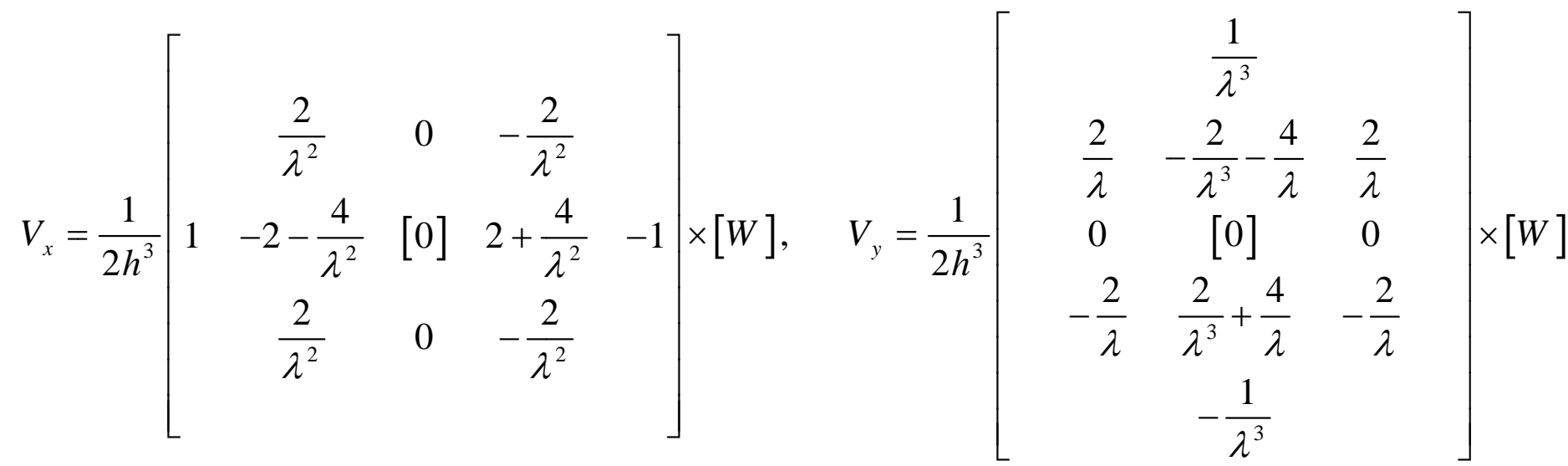

\subsubsection{Finite difference approximations in the vicinity of supported plate angles}

Additional nodes, which are outside the plate, are introduced in order to apply the governing equations at the boundary nodes and to satisfy the boundary conditions. The number of additional nodes corresponds to the number of boundary conditions at the node of interest: therefore, three additional nodes are introduced at a supported angle or at a free angle having a concentrated load acting on it, and four additional nodes at a free angle with no concentrated load acting on it. Figure 3 below is the case of a supported angle or a free angle with concentrated load acting on it; regular nodes on the plate edges (k; e; ee; eee; s; se; ss...) and additional nodes (nw; n; ne; w; sw...) are represented.

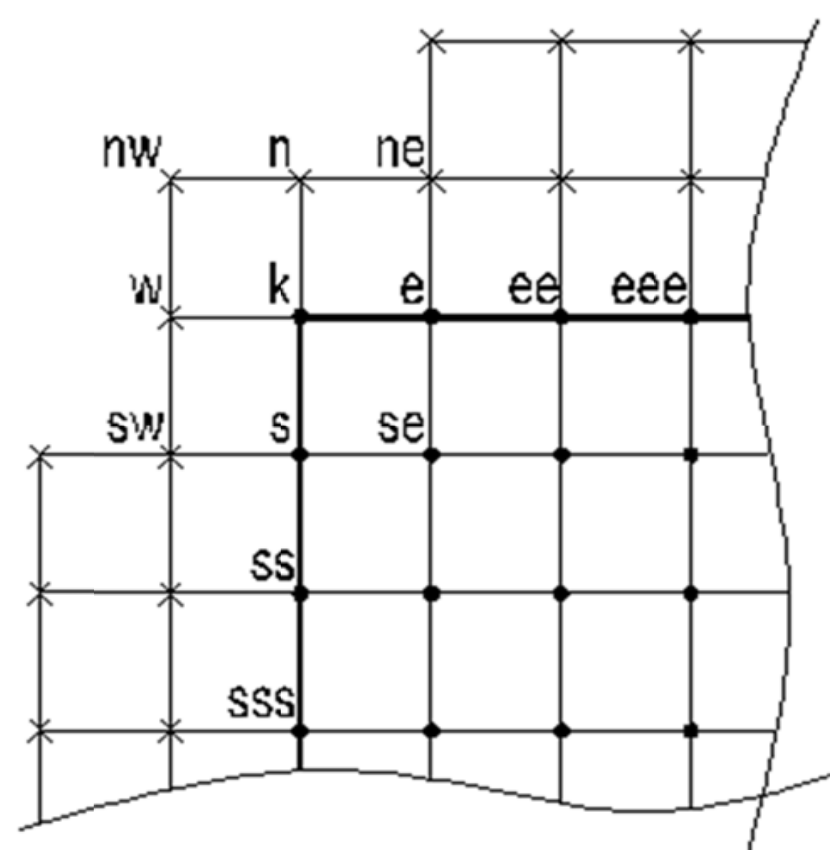

Figure 3 Supported plate angle with regular nodes $(\bullet)$ and additional nodes $(\times)$

Governing equation of the plate, bending moments, and twisting moments at node $k$

Equation (7h) is used for the bending terms $\partial^{4} \mathrm{w} / \partial \mathrm{x}^{4}$ and $\partial^{4} \mathrm{w} / \partial \mathrm{y}^{4}$ while Equation (9f) is used for the twisting term $\partial^{4} w / \partial x^{2} \partial y^{2}$. The governing equation (Equation (4)) of the plate at node $\mathrm{k}$ is then described with the following 13-point stencil 


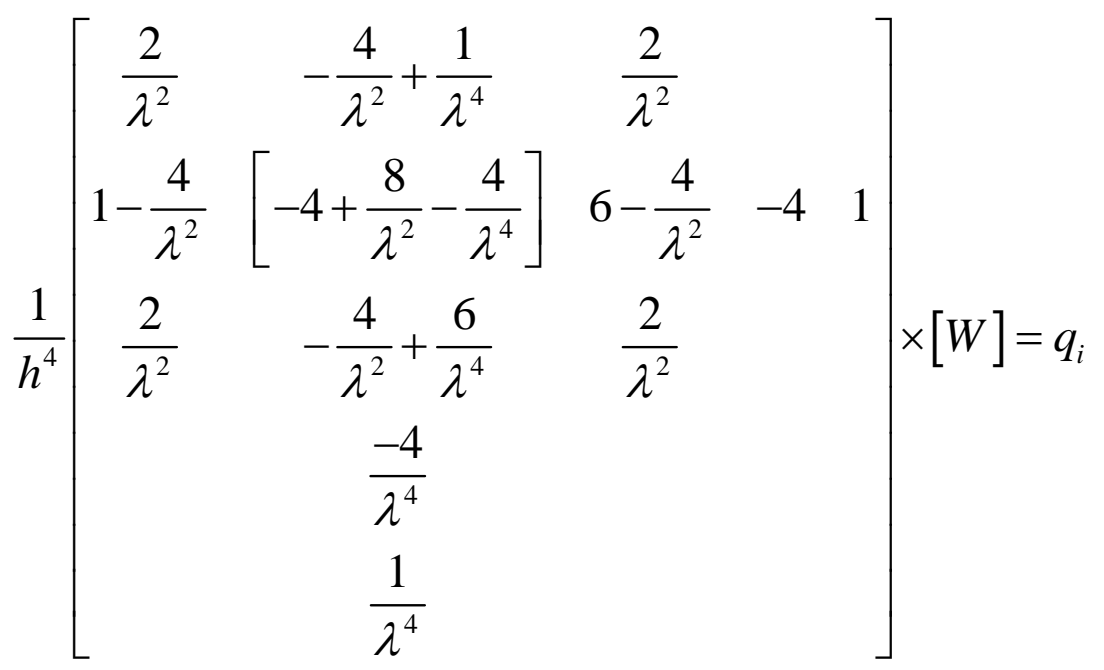

For other angle points of the plate, the governing equations are determined using the same principle. The bending and twisting moments per unit length, with Poisson's ratio $v=0$, are calculated using Equations (9i-k).

Governing equation, moments, and Kirchhoff shear forces at nodes e, ee, s, and ss The governing equation (Equation (4)) is described using the 13-point stencil of Equation (9g). The bending and twisting moments per unit length, and the Kirchhoff shear forces per unit length, with Poisson's ratio $v=0$, are calculated using Equations (9i-m).

\section{Governing equation, moments, and Kirchhoff shear forces at nodes eee and sss}

The 25-point stencil and the 13-point stencil can be considered as described in Section 2.1.2.1. In case of a 25-point stencil, the governing equation is formulated using Equation (8e); the bending/twisting moments per unit length, and the Kirchhoff shear forces per unit length, with Poisson's ratio $v=0$, are calculated using Equations (9a-e). In case of a 13point stencil, the governing equation is formulated using Equation (9g); the bending/twisting moments per unit length, and the Kirchhoff shear forces per unit length, with Poisson’s ratio $v=0$, are calculated using Equations (9i-m).

\subsubsection{Finite difference approximations in the vicinity of free plate angles}

Four additional nodes are introduced at the free plate angle, as represented in Figure 4. Alternatively, node nn could be considered instead of node ww, according to Figure 2. 


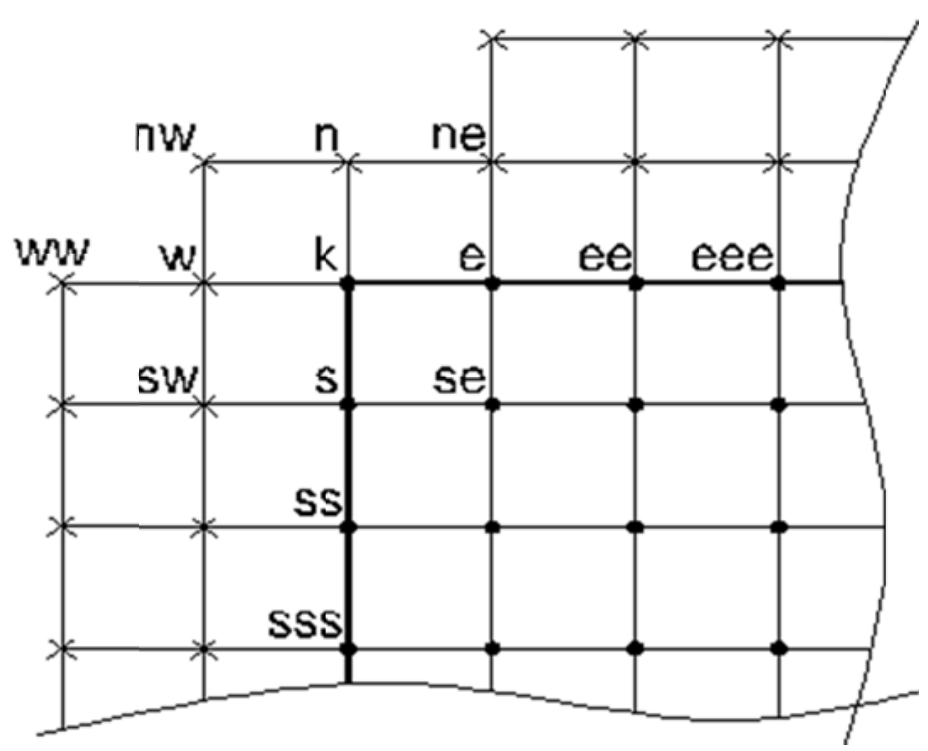

Figure 4 Free plate angle with regular nodes $(\bullet)$ and additional nodes $(\times)$

The analysis is conducted in the same manner as the case of supported plate angle.

\section{Governing equation, moments, and Kirchhoff shear forces at node $k$}

The governing equation at node $k$, Equation (4), is described with the following 13-point stencil

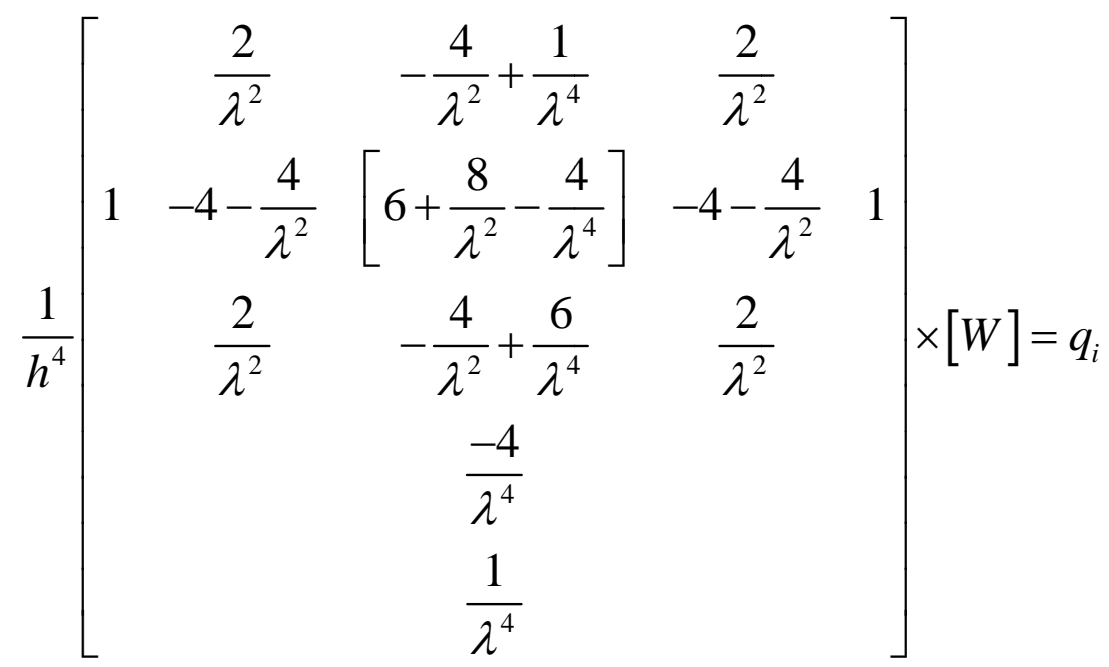

The bending and twisting moments per unit length at node $\mathrm{k}$ are determined using Equations (9i-k). The Kirchhoff shear force per unit length $V_{x}$ is determined using Equation (9l); $V_{y}$ at the other hand has following adjustment using the noncentered FDA of Equation (7g)) 


$$
V_{y}=\frac{1}{2 h^{3}}\left[\begin{array}{ccc}
\frac{2}{\lambda} & \frac{3}{\lambda^{3}}-\frac{4}{\lambda} & \frac{2}{\lambda} \\
0 & {\left[\frac{-10}{\lambda^{3}}\right]} & 0 \\
-\frac{2}{\lambda} & \frac{12}{\lambda^{3}}+\frac{4}{\lambda} & -\frac{2}{\lambda} \\
& \frac{-6}{\lambda^{3}} \\
& \frac{1}{\lambda^{3}}
\end{array}\right] \times[W]
$$

Governing equation, moments, and Kirchhoff shear forces at nodes e, ee, s, and ss The analysis is similar to that of Section 2.1.2.2.

Governing equation, moments, and Kirchhoff shear forces at nodes eee and sss

The analysis is conducted similarly to Section 2.1.2.2.

\subsubsection{Finite difference approximations at skew edges}

Figure 5 shows a skew edge with regular nodes and additional nodes. The outer normal $\mathbf{n}$ to the skew edge makes an angle $\alpha$ with the $+x$-axis. Two additional nodes are introduced at each edge node; therefore, governing equations can be applied at the edge nodes and boundary conditions be satisfied.

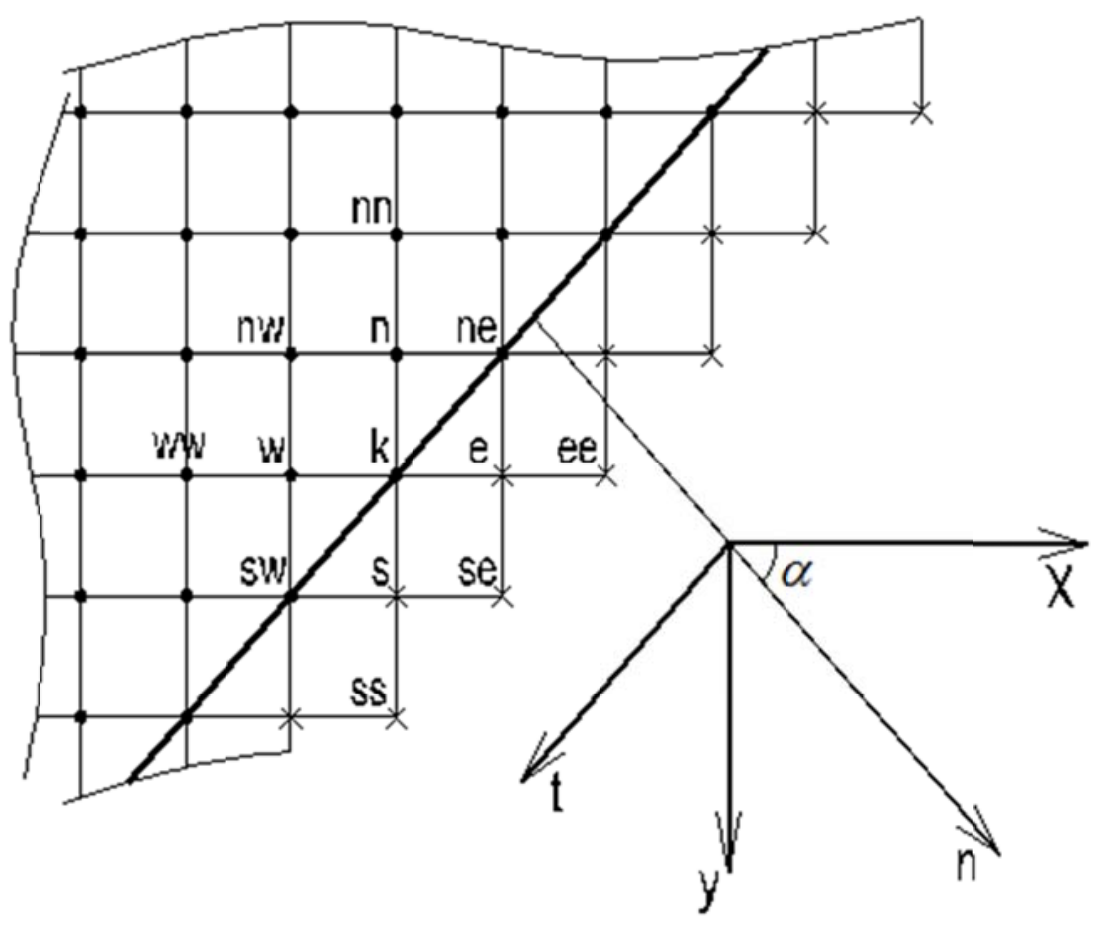

Figure 5 Plate skew edge with regular nodes $(\bullet)$ and additional nodes $(\times)$ 
The governing equation, FDA of Equation (4), at any edge node is expressed using the 13-point stencil of Equation (9g). The bending moments with respect to outer normal and tangential directions are expressed using Equations (1a-b), respectively, as follows

$$
m_{n}=-D \times\left(\frac{\partial^{2} w}{\partial n^{2}}+v \frac{\partial^{2} w}{\partial t^{2}}\right), \quad m_{t}=-D \times\left(\frac{\partial^{2} w}{\partial t^{2}}+v \frac{\partial^{2} w}{\partial n^{2}}\right)
$$

The slope $\partial \mathrm{w} / \partial \mathrm{n}$ of the deflection surface with respect to the outer normal direction, the curvatures $-\partial^{2} \mathrm{w} / \partial \mathrm{n}^{2}$ and $-\partial^{2} \mathrm{w} / \partial \mathrm{t}^{2}$ with respect to outer normal and tangential directions, respectively, are expressed using following formulas developed in Timoshenko et al. [11]

$$
\begin{aligned}
& \frac{\partial w}{\partial n}=\frac{\partial w}{\partial x} \cos \alpha+\frac{\partial w}{\partial y} \sin \alpha \\
& \frac{\partial^{2} w}{\partial n^{2}}=\frac{\partial^{2} w}{\partial x^{2}} \cos ^{2} \alpha+\frac{\partial^{2} w}{\partial x \partial y} \sin 2 \alpha+\frac{\partial^{2} w}{\partial y^{2}} \sin ^{2} \alpha \\
& \frac{\partial^{2} w}{\partial t^{2}}=\frac{\partial^{2} w}{\partial x^{2}} \sin ^{2} \alpha-\frac{\partial^{2} w}{\partial x \partial y} \sin 2 \alpha+\frac{\partial^{2} w}{\partial y^{2}} \cos ^{2} \alpha
\end{aligned}
$$

The partial derivatives $\partial \mathrm{w} / \partial \mathrm{x}$ and $\partial \mathrm{w} / \partial \mathrm{y}$ in Equation (14a) are expressed using Equations (7d) or (7j), $\partial^{2} \mathrm{w} / \partial \mathrm{x}^{2}$ and $\partial^{2} \mathrm{w} / \partial \mathrm{y}^{2}$ using Equation (7i), and $\partial^{2} \mathrm{w} / \partial \mathrm{x} \partial \mathrm{y}$ using Equation (7k).

The Kirchhoff shear force, with respect to the outer normal to the skew edge, is determined as follows

$$
V_{n}=V_{x} \cos \alpha+V_{y} \sin \alpha
$$

where $\mathrm{V}_{\mathrm{x}}$ and $\mathrm{V}_{\mathrm{y}}$ are Kirchhoff shear forces with respect to Cartesian coordinates, expressed using Equations (9l-m). Equations (13a), (14a-c), and (15) are needed to satisfy the boundary conditions at the skew edge.

\subsubsection{Finite difference approximations of loadings}

Let us determine here the FDA of the transverse load per unit area in the case of a varying distributed load q(x,y). Let $\left(\mathrm{x}_{\mathrm{i}}, \mathrm{y}_{\mathrm{i}}\right)$ be the Cartesian coordinates of node $\mathrm{i}$. This FDA, denoted by $\mathrm{q}_{\mathrm{i}}$, can be taken as the average load around the node of interest and is then expressed as follows:

$$
q_{i}=\frac{1}{\Delta x \Delta y} \iint q(x, y) d x d y
$$




\subsubsection{Analysis at positions of discontinuity}

Positions of discontinuity are positions of application of concentrated external loads (linearly distributed forces or moments, concentrated forces or moments), supports, hinges, springs, abrupt change of cross section, and change of grid spacing.

\subsubsection{Concentrated force $P$ at node $i$}

The concentrated load can be converted into a transverse load per unit area $\mathrm{q}_{\mathrm{i}}$ as follows

$$
q_{i}=\frac{P}{\Delta x \Delta y}
$$

where $\Delta \mathrm{x}$ and $\Delta \mathrm{y}$ are the grid spacing in $\mathrm{x}$ - and $\mathrm{y}$ - directions, respectively. The governing equation, FDA of Equation (4), is applied. However a more accurate approach, at the expense of intensive calculations, would be a local grid refinement; this will be carried out in a subsequent section.

\subsubsection{Linearly distributed forces or moments, linear supports/hinges}

Linearly distributed forces and moments can be converted into concentrated forces and couples of forces, respectively, and analyzed according to Section 2.1.3.1. Another approach, more accurate, is presented hereafter.

Let linearly distributed forces (p) and moments ( $\mathrm{m}^{*}$, positive counterclockwise) be applied on the plate. The loading is assumed parallel to Cartesian y-direction through the node i, as represented in Figure 6, and the grid spacings apart from the loading are $h_{k}$ and $h_{p}$. A loading parallel to Cartesian $x$-direction would be analyzedl similarly.

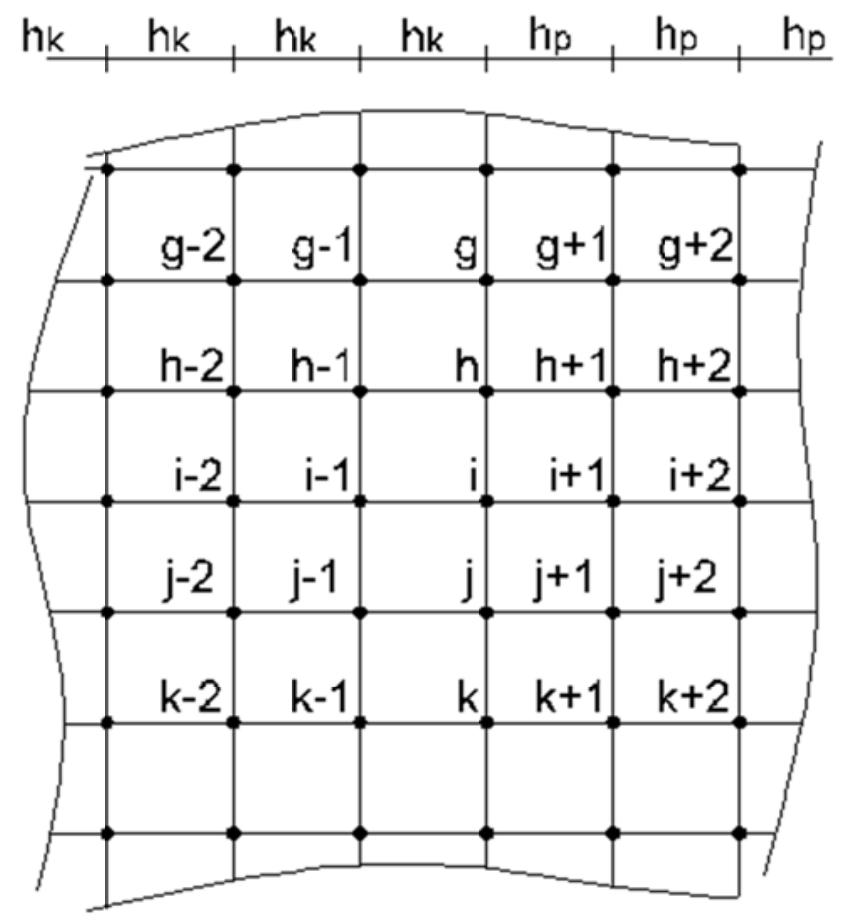

Figure 6 Plate subjected to linearly distributed forces and moments 
The model developed in this paper consists of realizing an opening of the plate along the linearly distributed loading (nodes ...g, h, i, j, k ...) and introducing additional nodes $(\times)$ in the opening, as represented in Figure 7a, b.
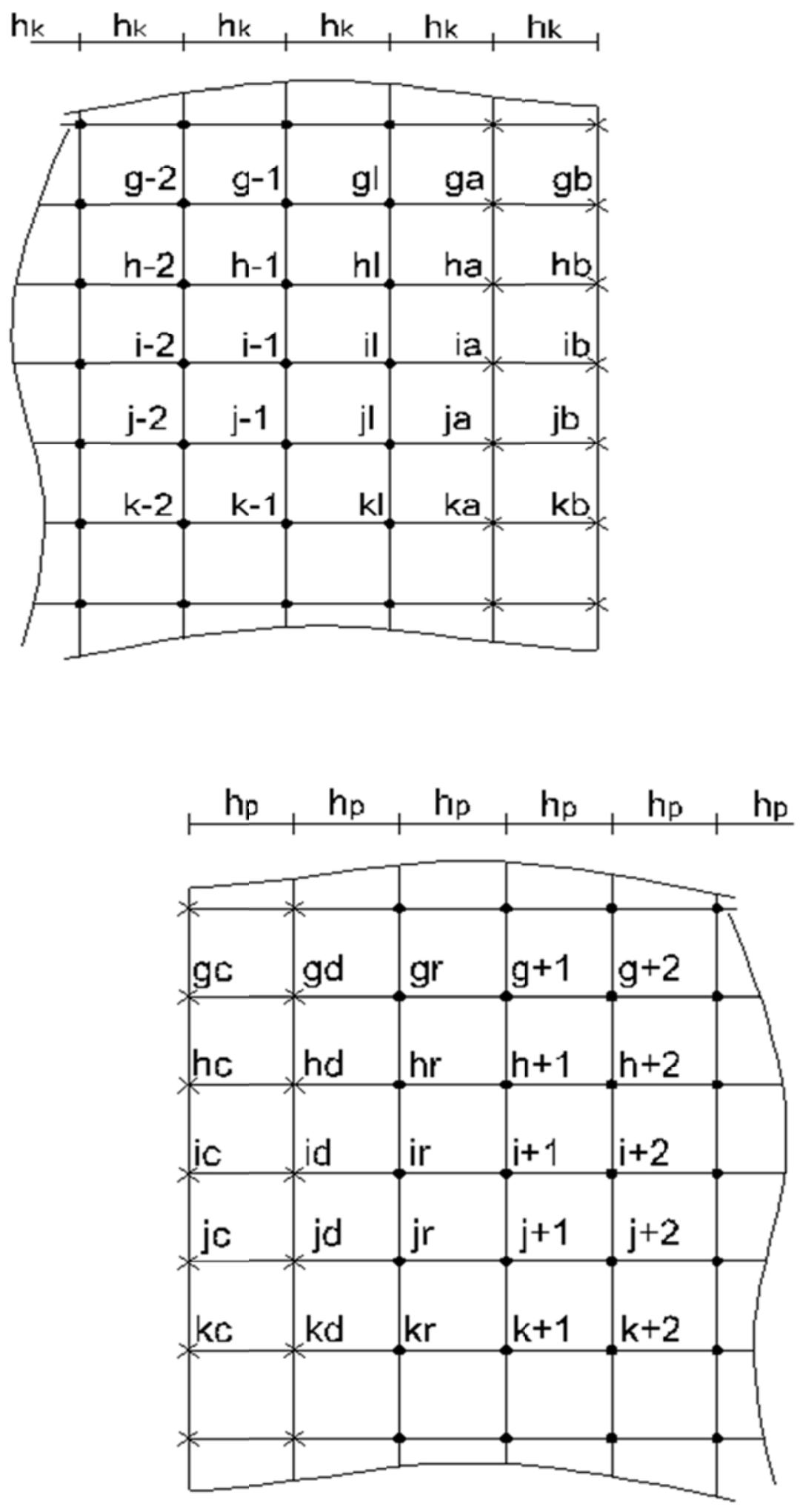

Figure 7a, b Opening of the plate and introduction of additional nodes $(\times)$ on the left side $(7 a)$ and right side (7b) 
The governing equation, FDA of Equation (4), is applied at any regular node $(\bullet)$ of the plate. While applying the governing equation to the first two nodes at each end of the loading, the FDA of the twisting term will be formulated as follows

$$
\frac{\partial^{4} w}{\partial x^{2} \partial y^{2}}=\frac{\partial^{2}}{\partial y^{2}}\left(\frac{\partial^{2} w}{\partial x^{2}}\right)
$$

whereby the partial derivatives with respect to y will have non-centered FDAs according to Equation (7f).

The continuity equations (Equations (19a-d) at node i, with Poisson's ratio $v=0$, express the continuity of the deflection and slope with respect to $\mathrm{x}$-axis, and the equilibrium of the bending moment $\mathrm{m}_{\mathrm{xx}}$ and Kirchhoff shear force $\mathrm{V}_{\mathrm{x}}$. In the case of a 25-point stencil the slope with respect to $\mathrm{x}$-axis, the bending moment $\mathrm{m}_{\mathrm{xx}}$ and the Kirchhoff shear force $\mathrm{V}_{\mathrm{x}}$ are expressed using Equations (7d), (9a), and (9d), respectively, and in the case of a 13-point stencil they are expressed using Equations (7j), (9i), and (9l), respectively. Equations (19b-c) are formulated for the case of a 25-point stencil.

$$
\begin{aligned}
& W_{i l}=W_{i r} \rightarrow W_{i l}=W_{i r} \\
& D \times \varphi_{x, i l}=D \times \varphi_{x, i r} \rightarrow \frac{W_{i-2}-8 W_{i-1}+8 W_{i a}-W_{i b}}{12 h_{k}}=\frac{W_{i c}-8 W_{i d}+8 W_{i+1}-W_{i+2}}{12 h_{p}} \\
& m_{x x, i l}-m_{x x, i r}=m^{*} \rightarrow \\
& \frac{W_{i-2}-16 W_{i-1}+30 W_{i l}-16 W_{i a}+W_{i b}}{12 h_{k}^{2}}-\frac{W_{i c}-16 W_{i d}+30 W_{i r}-16 W_{i+1}+W_{i+2}}{12 h_{p}{ }^{2}}=m^{*} \\
& V_{x, i l}-V_{x, i r}=p
\end{aligned}
$$

The derivatives with respect to y will have non-centered FDAs while applying Equation (19c-d) to the first two nodes at each end of the loading; Equations (7f) and (7i) will be then considered.

An adjustment of the continuity equations is made in case of a linear hinge (no continuity of the slope, $\mathrm{m}_{\mathrm{xx}, \mathrm{il}}=\mathrm{m}_{\mathrm{xx}, \mathrm{ir}}=0$ ), a linear support $\left(\mathrm{W}_{\mathrm{il}}=\mathrm{W}_{\mathrm{ir}}=0\right.$, no equation (19d)), or a spring.

\subsubsection{Monolithically connected beams or stiffeners}

A beam in y-direction is assumed monolithically connected to the plate, its torsion stiffness being considered or not. Let linearly distributed forces (p) and moments ( $\mathrm{m}^{*}$, positive counterclockwise) be applied on the beam.

\section{Beam without torsion stiffness}

The model represented in Figure 7a, b is considered. The beam deflections are denoted by $\mathrm{w}_{\mathrm{b}}$. Equations (19a-c) are applied; in addition, the following equations are set

$$
\begin{aligned}
& w_{b i}=w_{i l} \\
& E I_{b} \frac{d^{4} w_{b}}{d y^{4}}=p+V_{x, i r}-V_{x, i l}
\end{aligned}
$$


where $\mathrm{EI}_{\mathrm{b}}$ is the bending stiffness of the beam. Two boundary conditions must be satisfied at each beam's end; therefore, two additional nodes are introduced at each beam's end, with the deflections as unknowns. The term $\mathrm{d}^{4} \mathrm{w}_{\mathrm{b}} / \mathrm{dy}^{4}$ is expressed using Equation (8b).

\section{Beam with Saint Venant torsion stiffness}

The angles of twist of the beam are denoted by $\theta_{\mathrm{b}}$. Equations (19a-b) and (20a-b) are applied; in addition, the following equations are set

$$
\begin{aligned}
& \theta_{b i}=-\varphi_{x, i l} \rightarrow D \times \theta_{b i}=-\frac{W_{i-2}-8 W_{i-1}+8 W_{i a}-W_{i b}}{12 h_{k}} \\
& G I_{t} \frac{d^{2} \theta_{b}}{d y^{2}}=m_{x, i l}-m_{x, i r}-m^{*}
\end{aligned}
$$

where $\mathrm{GI}_{\mathrm{t}}$ is the torsional stiffness of the beam. Two boundary conditions on bending and one boundary condition on torsion must be satisfied at each beam's end; therefore, two additional nodes are introduced at each beam's end, one of which having two unknowns (deflection and angle of twist) and the other one unknown (deflection). The term $\mathrm{d}^{2} \theta_{\mathrm{b}} / \mathrm{dy}^{2}$ is expressed using Equation (7i).

\section{Beam with Saint Venant torsion stiffness and warping torsion stiffness}

Equations (19a-b), (20a-b), and (21a) are applied; in addition, the following equation is set

$$
E I_{\omega \omega} \frac{d^{4} \theta_{b}}{d y^{4}}-G I_{t} \frac{d^{2} \theta_{b}}{d y^{2}}=m^{*}+m_{x, i r}-m_{x, i l}
$$

where $\mathrm{EI}_{\omega \omega}$ is the warping torsion stiffness of the beam. Two boundary conditions on bending and two boundary conditions on torsion must be satisfied at each beam's end; therefore, two additional nodes are introduced at each beam's end, both of which having two unknowns (deflection and angle of twist). The term $d^{4} \theta_{b} / d y^{4}$ and $d^{2} \theta_{b} / d y^{2}$ are expressed using Equations (7a) and (7c), respectively.

\subsubsection{Concentrated support or spring at node $i$}

The unknown support reaction $S_{i}$ can be converted into a transverse load per unit area $q_{i}$ as follows

$$
q_{i}=-\frac{S_{i}}{\Delta x \Delta y}
$$


where $\Delta \mathrm{x}$ and $\Delta \mathrm{y}$ are the grid spacing in $\mathrm{x}$ - and $\mathrm{y}$ - directions, respectively. So, there is one unknown $\left(\mathrm{S}_{\mathrm{i}}\right)$ more and one more boundary condition (the deflection at node i is zero). As mentioned in Section 2.1.3.1, a local grid refinement would deliver more accurate results at the expense of intensive calculations. The case of a concentrated spring support with stiffness $\mathrm{K}$ is analyzed similarly, as follows

$$
q_{i}=-\frac{K w_{i}}{\Delta x \Delta y}
$$

\subsubsection{Elastic Winkler foundation over an area of the plate}

Given an elastic Winkler foundation having the stiffness $\mathrm{k}_{\mathrm{w}}$. Equation (4) becomes

$$
\frac{\partial^{4} w(x, y)}{\partial x^{4}}+2 \frac{\partial^{4} w(x, y)}{\partial x^{2} \partial y^{2}}+\frac{\partial^{4} w(x, y)}{\partial y^{4}}=\frac{q(x, y)-k_{W} w(x, y)}{D}
$$

To account for the elastic Winkler foundation, $\mathrm{k}_{\mathrm{Wh}}{ }^{4} / \mathrm{D}$ is added to the term associated to the node of interest in the stencil describing the governing equations (Equations (8e), (9g), (10), and (11)). Equation (8e) i.e. becomes

$\frac{1}{h^{4}}\left[\begin{array}{ccccc}\frac{1}{72 \lambda^{2}} & \frac{-2}{9 \lambda^{2}} & \frac{1}{\lambda^{4}}+\frac{5}{12 \lambda^{2}} & \frac{-2}{9 \lambda^{2}} & \frac{1}{72 \lambda^{2}} \\ \frac{-2}{9 \lambda^{2}} & \frac{32}{9 \lambda^{2}} & \frac{-4}{\lambda^{4}}-\frac{20}{3 \lambda^{2}} & \frac{32}{9 \lambda^{2}} & \frac{-2}{9 \lambda^{2}} \\ 1+\frac{5}{12 \lambda^{2}} & -4-\frac{20}{3 \lambda^{2}} & 6+\frac{6}{\lambda^{4}}+\frac{25}{2 \lambda^{2}}+\frac{k_{W} h^{4}}{D} & -4-\frac{20}{3 \lambda^{2}} & 1+\frac{5}{12 \lambda^{2}} \\ \frac{-2}{9 \lambda^{2}} & \frac{32}{9 \lambda^{2}} & \frac{-4}{\lambda^{4}}-\frac{20}{3 \lambda^{2}} & \frac{32}{9 \lambda^{2}} & \frac{-2}{9 \lambda^{2}} \\ \frac{1}{72 \lambda^{2}} & \frac{-2}{9 \lambda^{2}} & \frac{1}{\lambda^{4}}+\frac{5}{12 \lambda^{2}} & \frac{-2}{9 \lambda^{2}} & \frac{1}{72 \lambda^{2}}\end{array}\right] \times[W]=q_{i}$

\subsubsection{Local grid refinement}

A local grid refinement can be considered at positions where concentrated load or bending moment is applied, or at a concentrated support or spring. Refinement nodes (o) are then introduced around the node of interest, as represented in Figure 8 . 


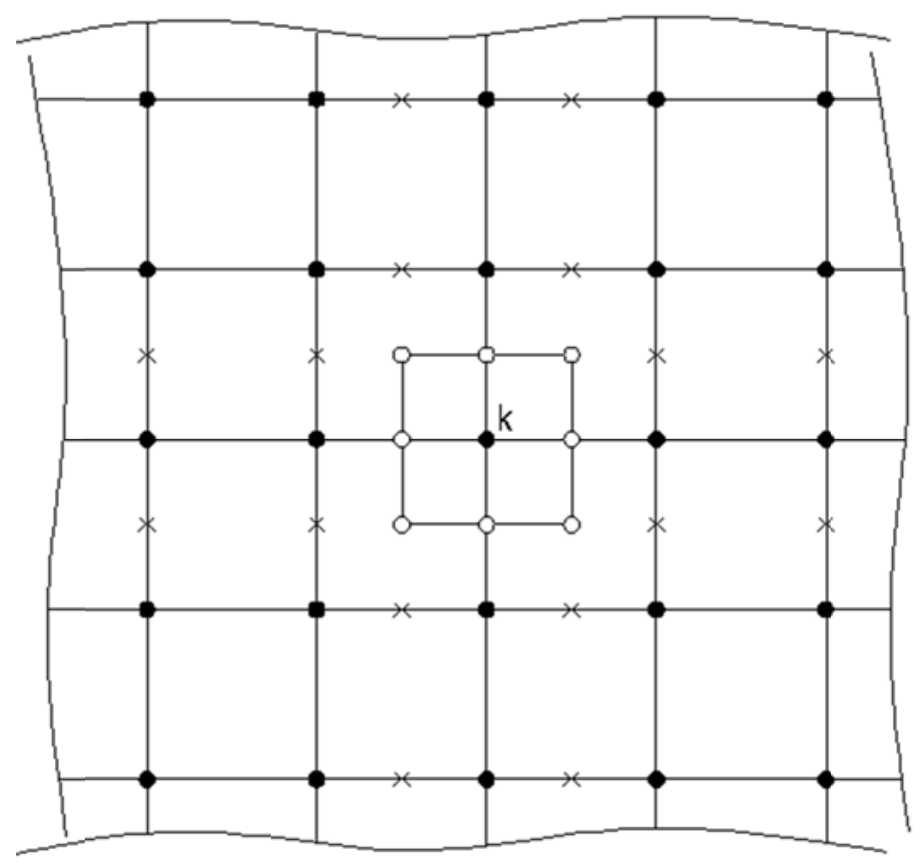

Figure 8 Local grid refinement with regular nodes $(\bullet)$, refinement nodes (o), additional nodes $(\times)$

Governing equations are applied at regular nodes (•) and at refinement nodes (o). However, Lagrange interpolating polynomials (Equations $(5,6)$ ) would have to be considered since the grid spacings are not constant in the vicinity of the point of interest. Additional nodes $(\times)$ are needed for the FDAs of the governing equations (Equation (4)), whereby the deflections and second derivatives of the deflection surface at additional nodes are obtained using Lagrange interpolating polynomials.

\subsection{Second-order analysis of a Kirchhoff plate}

\subsubsection{Governing equations}

The second-order analysis of an isotropic Kirchhoff plate is governed by the following requation

$$
\frac{\partial^{4} w}{\partial x^{4}}+2 \frac{\partial^{4} w}{\partial x^{2} \partial y^{2}}+\frac{\partial^{4} w}{\partial y^{4}}=\frac{1}{D}\left(q+N_{x} \frac{\partial^{2} w}{\partial x^{2}}+N_{y} \frac{\partial^{2} w}{\partial y^{2}}+2 N_{x y} \frac{\partial^{2} w}{\partial x \partial x}\right)
$$

where $\mathrm{N}_{\mathrm{x}}$ and $\mathrm{N}_{\mathrm{y}}$ are axial forces (positive in tension) per unit length in $\mathrm{x}$ - and $\mathrm{y}$ - direction, respectively, and $\mathrm{N}_{\mathrm{xy}}$ is the shearing force per unit length. Equation (27) has been derived by Saint Venant in his translation of Clebsch [12], “Théorie de l'élasticité des corps solides.” 
The bending moments per unit length $\mathrm{m}_{\mathrm{xx}}$ and $\mathrm{m}_{\mathrm{yy}}$, and the twisting moments per unit length $\mathrm{m}_{\mathrm{xy}}$, respectively, are expressed using Equations (1a-c). The transverse forces $\mathrm{T}$ per unit length are related to the Kirchhoff shear forces per unit length $\mathrm{V}$ as follows:

$$
T_{x}=V_{x}+N_{x} \frac{\partial w}{\partial x}, \quad T_{y}=V_{y}+N_{y} \frac{\partial w}{\partial y}
$$

\subsubsection{Finite difference approximations for an isotropic Kirchhoff-Love plate}

\subsubsection{Finite difference approximations at an interior node}

Let the nodes spacings be $\Delta \mathbf{x}=\mathbf{h}$ and $\Delta \mathbf{y}=\lambda \mathbf{h}$ in $\mathrm{x}$ - and y-direction, respectively. Following parameters are defined

$$
\gamma_{x}=\frac{N_{x} h^{2}}{D}, \quad \gamma_{y}=\frac{N_{y} h^{2}}{D}, \quad \gamma_{x y}=\frac{N_{x y} h^{2}}{D}
$$

Similarly to Section 2.1.2.1, the analysis is conducted considering a 25-point stencil and a 13-point stencil.

Governing equation, moments, and Kirchhoff shear forces using a 25-point stencil

Applying Equations (7c-d), (8a-d), and (29a-c) in Equation (27) yields the stencil notation of the governing equation (Equation (27)) of the plate as follows

$$
\frac{1}{h^{4}}\left[\begin{array}{ccccc}
\frac{1}{72 \lambda^{2}}-\frac{\gamma_{x y}}{72 \lambda} & \frac{-2}{9 \lambda^{2}}+\frac{\gamma_{x y}}{9 \lambda} & \frac{1}{\lambda^{4}}+\frac{5}{12 \lambda^{2}}+\frac{\gamma_{y}}{12 \lambda^{2}} & \frac{-2}{9 \lambda^{2}}-\frac{\gamma_{x y}}{9 \lambda} & \frac{1}{72 \lambda^{2}}+\frac{\gamma_{x y}}{72 \lambda} \\
\frac{-2}{9 \lambda^{2}}+\frac{\gamma_{x y}}{9 \lambda} & \frac{32}{9 \lambda^{2}}-\frac{8 \gamma_{x y}}{9 \lambda} & \frac{-4}{\lambda^{4}}-\frac{20}{3 \lambda^{2}}-\frac{4 \gamma_{y}}{3 \lambda^{2}} & \frac{32}{9 \lambda^{2}}+\frac{8 \gamma_{x y}}{9 \lambda} & \frac{-2}{9 \lambda^{2}}-\frac{\gamma_{x y}}{9 \lambda} \\
1+\frac{5}{12 \lambda^{2}}+\frac{\gamma_{x}}{12} & -4-\frac{20}{3 \lambda^{2}}-\frac{4 \gamma_{x}}{3} & {\left[\begin{array}{c}
6+\frac{6}{\lambda^{4}}+\frac{25}{2 \lambda^{2}}+ \\
\frac{5 \gamma_{x}}{2}+\frac{5 \gamma_{y}}{2 \lambda^{2}}
\end{array}\right]} & -4-\frac{20}{3 \lambda^{2}}-\frac{4 \gamma_{x}}{3} & 1+\frac{5}{12 \lambda^{2}}+\frac{\gamma_{x}}{12} \\
\frac{-2}{9 \lambda^{2}}-\frac{\gamma_{x y}}{9 \lambda} & \frac{32}{9 \lambda^{2}}+\frac{8 \gamma_{x y}}{9 \lambda} & \frac{-4}{\lambda^{4}}-\frac{20}{3 \lambda^{2}}-\frac{4 \gamma_{y}}{3 \lambda^{2}} & \frac{32}{9 \lambda^{2}}-\frac{8 \gamma_{x y}}{9 \lambda} & \frac{-2}{9 \lambda^{2}}+\frac{\gamma_{x y}}{9 \lambda} \\
\frac{1}{72 \lambda^{2}}+\frac{\gamma_{x y}}{72 \lambda} & \frac{-2}{9 \lambda^{2}}-\frac{\gamma_{x y}}{9 \lambda} & \frac{1}{\lambda^{4}}+\frac{5}{12 \lambda^{2}}+\frac{\gamma_{y}}{12 \lambda^{2}} & \frac{-2}{9 \lambda^{2}}+\frac{\gamma_{x y}}{9 \lambda} & \frac{1}{72 \lambda^{2}}-\frac{\gamma_{x y}}{72 \lambda}
\end{array}\right] \times[W]=q_{i}
$$


The bending/twisting moments are determined using Equations (9a-c), and the transverse forces using Equations (28ab), (9d-e), (7d), and (29a-b), as follows

$$
\begin{aligned}
& T_{x, k}=\frac{1}{2 h^{3}}\left[\begin{array}{ccccc}
\frac{1}{36 \lambda^{2}} & \frac{-2}{9 \lambda^{2}} & 0 & \frac{2}{9 \lambda^{2}} & \frac{-1}{36 \lambda^{2}} \\
\frac{-4}{9 \lambda^{2}} & \frac{32}{9 \lambda^{2}} & 0 & \frac{-32}{9 \lambda^{2}} & \frac{4}{9 \lambda^{2}} \\
1+\frac{5}{6 \lambda^{2}}+\frac{\gamma_{x}}{6} & -2-\frac{20}{3 \lambda^{2}}-\frac{4 \gamma_{x}}{3} & {[0]} & 2+\frac{20}{3 \lambda^{2}}+\frac{4 \gamma_{x}}{3} & -1-\frac{5}{6 \lambda^{2}}-\frac{\gamma_{x}}{6} \\
\frac{-4}{9 \lambda^{2}} & \frac{32}{9 \lambda^{2}} & 0 & \frac{-32}{9 \lambda^{2}} & \frac{4}{9 \lambda^{2}} \\
\frac{1}{36 \lambda^{2}} & \frac{-2}{9 \lambda^{2}} & 0 & \frac{2}{9 \lambda^{2}} & \frac{-1}{36 \lambda^{2}}
\end{array}\right] \times[W] \\
& T_{y, k}=\frac{1}{2 h^{3}}\left[\begin{array}{ccccc}
\frac{1}{36 \lambda} & \frac{-4}{9 \lambda} & \frac{1}{\lambda^{3}}+\frac{5}{6 \lambda}+\frac{\gamma_{y}}{6 \lambda} & \frac{-4}{9 \lambda} & \frac{1}{36 \lambda} \\
\frac{-2}{9 \lambda} & \frac{32}{9 \lambda} & -\frac{2}{\lambda^{3}}-\frac{20}{3 \lambda}-\frac{4 \gamma_{y}}{3 \lambda} & \frac{32}{9 \lambda} & \frac{-2}{9 \lambda} \\
0 & 0 & {[0]} & 0 & 0 \\
\frac{2}{9 \lambda} & \frac{-32}{9 \lambda} & \frac{2}{\lambda^{3}}+\frac{20}{3 \lambda}+\frac{4 \gamma_{y}}{3 \lambda} & \frac{-32}{9 \lambda} & \frac{2}{9 \lambda} \\
\frac{-1}{36 \lambda} & \frac{4}{9 \lambda} & -\frac{1}{\lambda^{3}}-\frac{5}{6 \lambda}-\frac{\gamma_{y}}{6 \lambda} & \frac{4}{9 \lambda} & \frac{-1}{36 \lambda}
\end{array}\right] \times[W]
\end{aligned}
$$

Governing equation, moments, and Kirchhoff shear forces using a 13-point stencil

The stencil notation of the governing equation (Equation (27)) of the plate is as follows

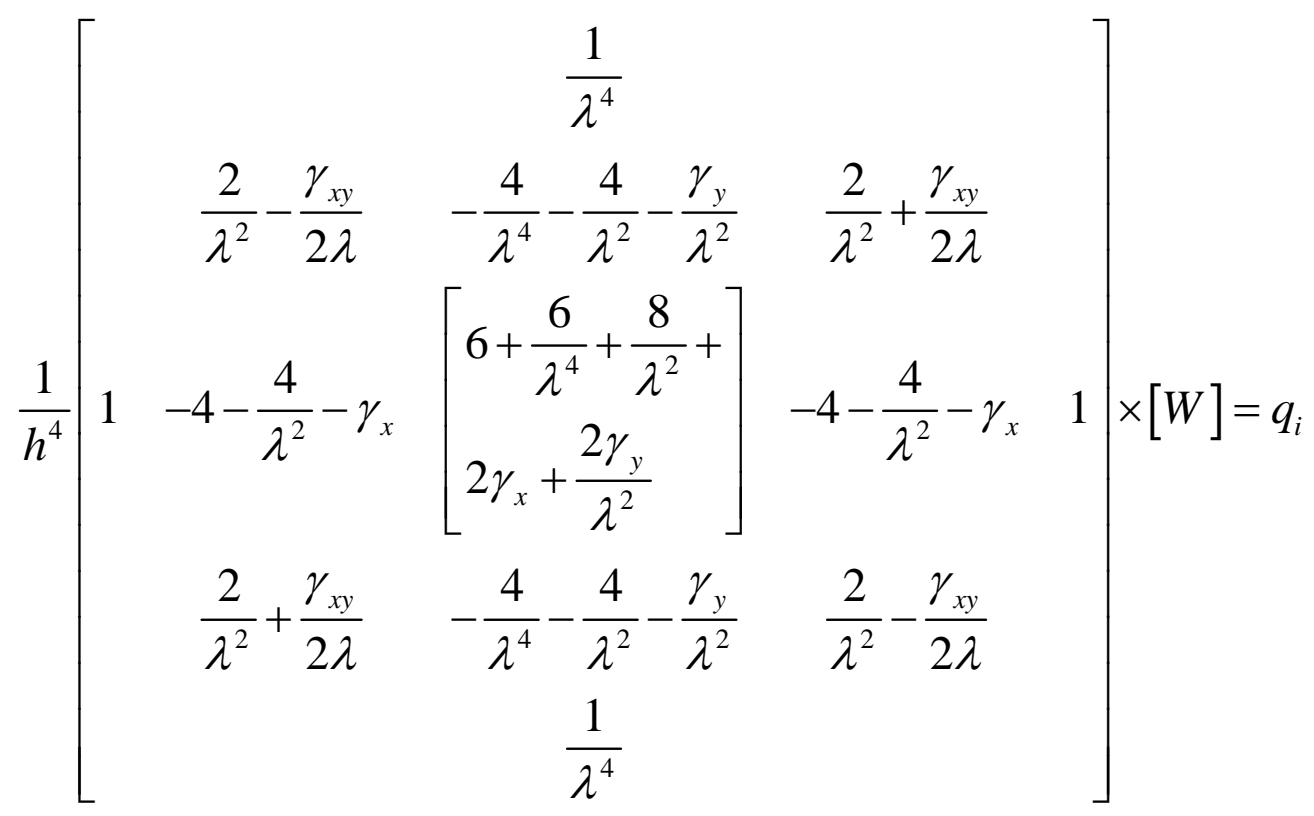


The bending/twisting moments per unit length are determined using Equations (9i-k), and the transverse forces per unit length using Equations (28a-b), (9l-m), (7j), and (29a-b), as follows
$T_{x}=\frac{1}{2 h^{3}}\left[\begin{array}{c}\frac{2}{\lambda^{2}} \\ 1-2-\frac{4}{\lambda^{2}}-\gamma_{x} \\ \frac{2}{\lambda^{2}}\end{array}\right.$
$0 \quad-\frac{2}{\lambda^{2}}$
$\left.\begin{array}{ll}-\frac{2}{\lambda^{2}} \\ \frac{4}{\lambda^{2}}+\gamma_{x} & -1 \\ -\frac{2}{\lambda^{2}}\end{array}\right] \times[W]$,
$\frac{1}{\lambda^{3}}$

2.2.2.2

Finite difference approximations in the vicinity of supported plate angles

The node distribution was represented in Figure 3.

Governing equation of the plate, bending moments, and twisting moments at node $k$

Applying the formulas developed in first-order analysis (Section 2.1.2.2) and Equations (29a-c) into Equation (27) yields the stencil notation of the governing equation (Equation (27)) of the plate as follows

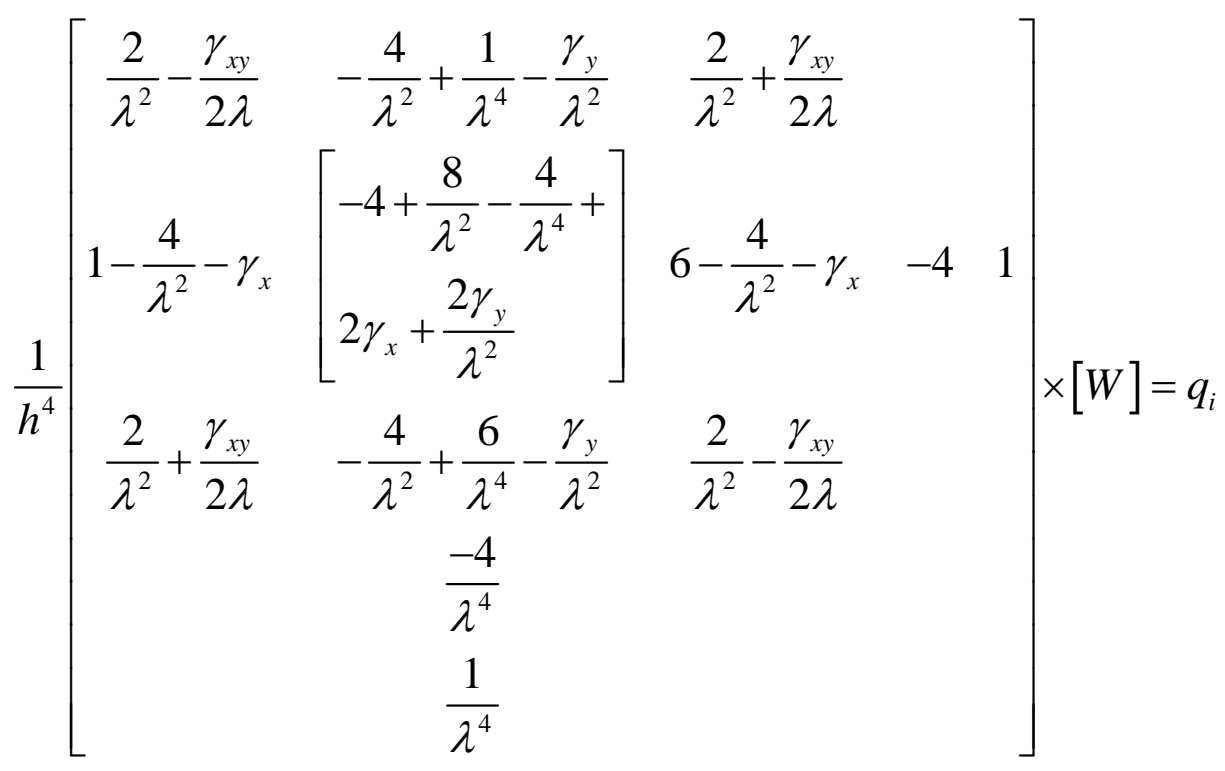

The bending/twisting moments are determined using Equations (9i-k).

Governing equation, moments, and Kirchhoff shear forces at nodes e, ee, s, and ss The governing equation (Equation (27)) is described using the 13-point stencil of Equation (32). The bending/twisting moments per unit length and the Kirchhoff transverse forces per unit length, with Poisson's ratio $v=0$, are calculated using Equations (9i-k) and (33), respectively. 


\section{Governing equation, moments, and Kirchhoff shear forces at nodes eee and sss}

The 25-point stencil and the 13-point stencil can be considered as described in Section 2.1.2.1. In case of a 25-point stencil, the governing equation is formulated with Equation (30); the bending/twisting moments per unit length, and the transverse forces per unit length, with Poisson's ratio $v=0$, are calculated using Equations (9a-c) and (31), respectively. In case of a 13-point stencil, the governing equation is formulated with Equation (32); the bending/twisting moments per unit length, and the transverse forces per unit length, with Poisson's ratio $v=0$, are calculated using Equations (9i-k) and (33), respectively.

\subsubsection{Finite difference approximations in the vicinity of free plate angles}

The node distribution was represented in Figure 4.

Governing equation of the plate, bending moments, and twisting moments at node $k$ Applying the formulas developed in first-order analysis (Section 2.1.2.3) and Equations (29a-c) into Equation (27) yields the stencil notation of the governing equation (Equation (27)) of the plate as follows

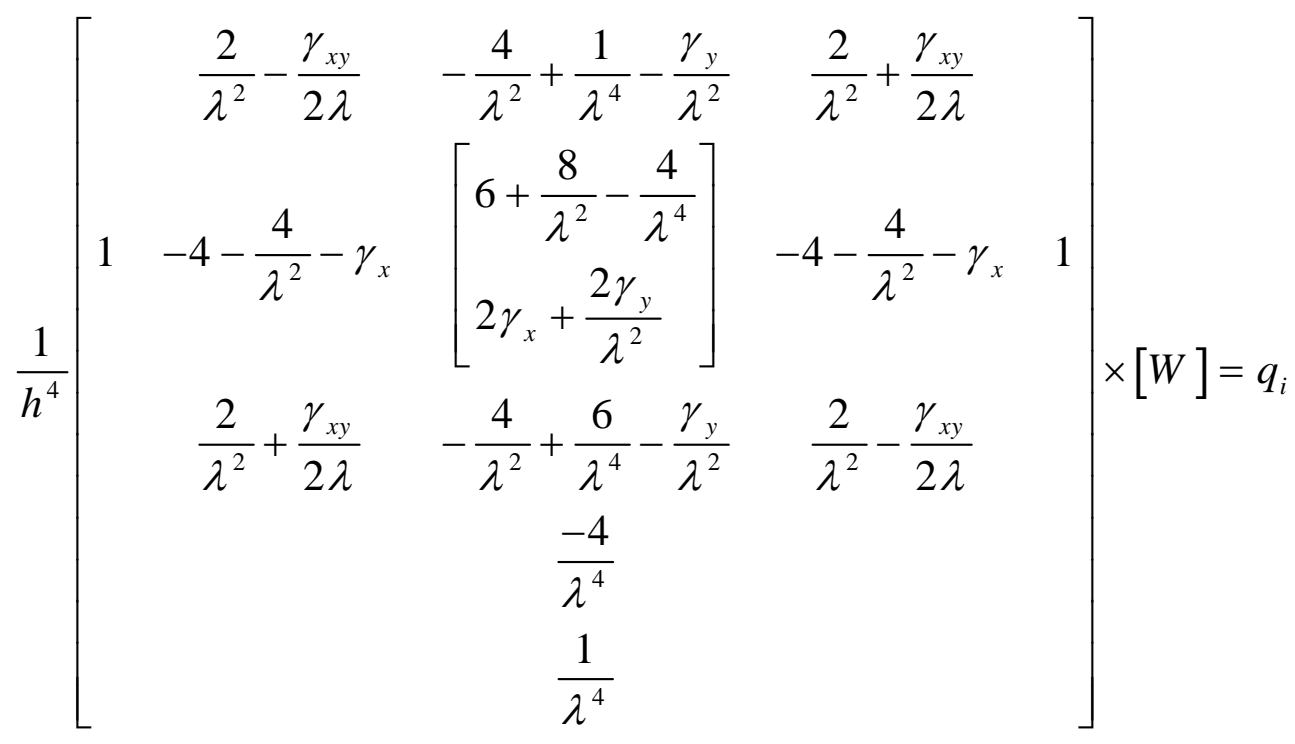

The bending/twisting moments per unit length are determined using Equations (9i-k). The transverse force per unit length $T_{x}$ is determined using Equation (33a), and $T_{y}$ using Equation (12), (28b), (29b), and (7j) as follows

$$
T_{y}=\frac{1}{2 h^{3}}\left[\begin{array}{ccc}
\frac{2}{\lambda} & \frac{3}{\lambda^{3}}-\frac{4}{\lambda}-\frac{\gamma_{y}}{\lambda} & \frac{2}{\lambda} \\
0 & {\left[\frac{-10}{\lambda^{3}}\right]} & 0 \\
-\frac{2}{\lambda} & \frac{12}{\lambda^{3}}+\frac{4}{\lambda}+\frac{\gamma_{y}}{\lambda} & -\frac{2}{\lambda} \\
\frac{-6}{\lambda^{3}} \\
\frac{1}{\lambda^{3}}
\end{array}\right] \times[W]
$$


Governing equation, moments, and Kirchhoff shear forces at nodes e, ee, s, and ss

The governing equation, Equation (27), is given by Equation (32). The bending/twisting moments per unit length and the transverse forces per unit length are determined using Equations (9i-k) and (33), respectively.

Governing equation, moments, and Kirchhoff shear forces at nodes eee and sss

The dispositions of Section 2.2.2.2 applied.

\subsubsection{Finite difference approximations at skew edges}

The node distribution of Section 2.1.2.3 applied. The governing equation, FDA of Equation (27), at any edge node is expressed using the 13-point stencil of Equation (32). The slope $\partial \mathrm{w} / \partial \mathrm{n}$ of the deflection surface with respect to the outer normal direction and the bending moments with respect to outer normal and tangential directions are expressed similarly to Section 2.1.2.3.

The transverse force $T_{n}$, with respect to the outer normal to the skew edge, is determined as follows

$$
T_{n}=T_{x} \cos \alpha+T_{y} \sin \alpha
$$

where $T_{x}$ and $T_{y}$ are transverse forces with respect to Cartesian coordinates, expressed using Equations (33).

\subsubsection{Analysis at positions of discontinuity}

The dispositions of Section 2.2.2 applied; however the Kirchhoff shear forces are replaced by the transverse forces.

\subsection{Vibration analysis of an isotropic Kirchhoff-Love plate}

\subsubsection{Free vibration analysis of a plate with constant thickness}

Our focus here is to determine the eigenfrequencies of the plate. Damping is not considered. A second-order analysis is conducted; the first-order analysis can be deduced. The deflection surface is denoted by $\mathrm{w}^{*}(\mathrm{x}, \mathrm{y}, \mathrm{t})$. The governing equation can be expressed as follows:

$$
\begin{aligned}
& \frac{\partial^{4} w^{*}(x, y, t)}{\partial x^{4}}+2 \frac{\partial^{4} w^{*}(x, y, t)}{\partial x^{2} \partial y^{2}}+\frac{\partial^{4} w^{*}(x, y, t)}{\partial y^{4}}= \\
& \frac{1}{D}\left(N_{x} \frac{\partial^{2} w^{*}(x, y, t)}{\partial x^{2}}+N_{y} \frac{\partial^{2} w^{*}(x, y, t)}{\partial y^{2}}+2 N_{x y} \frac{\partial^{2} w^{*}(x, y, t)}{\partial x \partial x}-\rho d \frac{\partial^{2} w^{*}(x, y, t)}{\partial t^{2}}\right)
\end{aligned}
$$

where $\rho$ is the plate's mass per unit volume and $d$ is the plate thickness. A harmonic vibration being assumed, $\mathrm{w}^{*}(\mathrm{x}, \mathrm{y}, \mathrm{t})$ can be expressed as follows:

$$
w^{*}(x, y, t)=w(x, y) \times \sin (\omega t+\theta)
$$


Here, $\omega$ is the circular frequency of the plate. Substituting Equation (39a) into Equation (38) yields

$$
\frac{\partial^{4} w}{\partial x^{4}}+2 \frac{\partial^{4} w}{\partial x^{2} \partial y^{2}}+\frac{\partial^{4} w}{\partial y^{4}}=\frac{1}{D}\left(q+N_{x} \frac{\partial^{2} w}{\partial x^{2}}+N_{y} \frac{\partial^{2} w}{\partial y^{2}}+2 N_{x y} \frac{\partial^{2} w}{\partial x \partial x}+\rho d \omega^{2} w(x)\right)
$$

The FDAs of Equation (39b) are obtained by adding - $\rho d \omega^{2} h^{4} / D$ to the term associated to the node of interest in the stencil describing the governing equations. Therefore, Equation (30) i.e. becomes

$$
\frac{1}{h^{4}}\left[\begin{array}{ccccc}
\frac{1}{72 \lambda^{2}}-\frac{\gamma_{x y}}{72 \lambda} & \frac{-2}{9 \lambda^{2}}+\frac{\gamma_{x y}}{9 \lambda} & \frac{1}{\lambda^{4}}+\frac{5}{12 \lambda^{2}}+\frac{\gamma_{y}}{12 \lambda^{2}} & \frac{-2}{9 \lambda^{2}}-\frac{\gamma_{x y}}{9 \lambda} & \frac{1}{72 \lambda^{2}}+\frac{\gamma_{x y}}{72 \lambda} \\
\frac{-2}{9 \lambda^{2}}+\frac{\gamma_{x y}}{9 \lambda} & \frac{32}{9 \lambda^{2}}-\frac{8 \gamma_{x y}}{9 \lambda} & \frac{-4}{\lambda^{4}}-\frac{20}{3 \lambda^{2}}-\frac{4 \gamma_{y}}{3 \lambda^{2}} & \frac{32}{9 \lambda^{2}}+\frac{8 \gamma_{x y}}{9 \lambda} & \frac{-2}{9 \lambda^{2}}-\frac{\gamma_{x y}}{9 \lambda} \\
1+\frac{5}{12 \lambda^{2}}+\frac{\gamma_{x}}{12} & -4-\frac{20}{3 \lambda^{2}}-\frac{4 \gamma_{x}}{3} & {\left[\begin{array}{c}
6+\frac{6}{\lambda^{4}}+\frac{25}{2 \lambda^{2}}+ \\
\frac{5 \gamma_{x}}{2}+\frac{5 \gamma_{y}}{2 \lambda^{2}}-\frac{\rho d \omega^{2} h^{4}}{D}
\end{array}\right]} & -4-\frac{20}{3 \lambda^{2}}-\frac{4 \gamma_{x}}{3} & 1+\frac{5}{12 \lambda^{2}}+\frac{\gamma_{x}}{12} \\
\frac{-2}{9 \lambda^{2}}-\frac{\gamma_{x y}}{9 \lambda} & \frac{32}{9 \lambda^{2}}+\frac{8 \gamma_{x y}}{9 \lambda} & \frac{-4}{\lambda^{4}}-\frac{20}{3 \lambda^{2}}-\frac{4 \gamma_{y}}{3 \lambda^{2}} & \frac{32}{9 \lambda^{2}}-\frac{8 \gamma_{x y}}{9 \lambda} & \frac{-2}{9 \lambda^{2}}+\frac{\gamma_{x y}}{9 \lambda} \\
\frac{1}{72 \lambda^{2}}+\frac{\gamma_{x y}}{72 \lambda} & \frac{-2}{9 \lambda^{2}}-\frac{\gamma_{x y}}{9 \lambda} & \frac{1}{\lambda^{4}}+\frac{5}{12 \lambda^{2}}+\frac{\gamma_{y}}{12 \lambda^{2}} & \frac{-2}{9 \lambda^{2}}+\frac{\gamma_{x y}}{9 \lambda} & \frac{1}{72 \lambda^{2}}-\frac{\gamma_{x y}}{72 \lambda}
\end{array}\right] \times[W]=0
$$

The bending/twisting moments per unit length, the shear forces and transversal forces per unit length are calculated similarly to previous sections.

\section{Effect of a concentrated mass}

We analyzed the dynamic behavior of a plate carrying a concentrated mass at node $i$; the mass $M_{p}$ is defined as follows

$$
M_{p}=m_{p} \times \rho \times \Delta x \times \Delta y \times d
$$

where $\mathrm{m}_{\mathrm{p}}$ is the dimensionless mass, and $\Delta \mathrm{x}$ and $\Delta \mathrm{y}=\lambda \Delta \mathrm{x}$ are the node spacings in $\mathrm{x}$ - and $\mathrm{y}$-direction, respectively.

The FDA of the governing equation at node $i$ is obtained by adding $-m_{p} \rho d \omega^{2} h^{4} / D$ to the term associated to the node of interest in the stencil. 
Effect of a spring-mass system: We analyzed the dynamic behavior of a plate carrying a spring-mass system at node i, as represented in Figure 9.

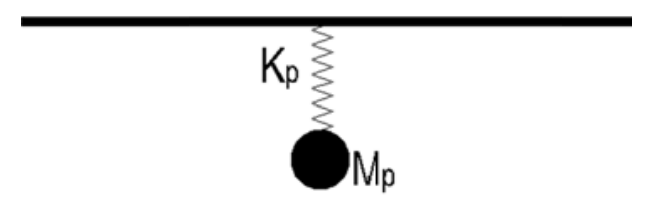

Figure 9 Vibration of a plate carrying a spring-mass system

The deflection of the mass was denoted by $\mathrm{w}_{\mathrm{iM}}$. The concentrated mass $\mathrm{M}_{\mathrm{p}}$ is defined according to Equation (41), and the stiffness $K_{p}$ of the spring is defined as follows,

$$
K_{p}=k_{p} \times \frac{D}{\Delta x \times \Delta y}
$$

where $\mathrm{k}_{\mathrm{p}}$ is the dimensionless spring stiffness. The FDA of the governing equation at node $\mathrm{i}$ is obtained by setting $\mathrm{m}_{\mathrm{p}} \rho \mathrm{d} \omega^{2} / \mathrm{D} \times \mathrm{W}_{\mathrm{iM}}$ at the right-hand side of Equation (40) instead of zero. In addition, following equilibrium equation is set

$$
\left(m_{p} \lambda^{2} \frac{\rho d \omega^{2} h^{4}}{D}-k_{p}\right) W_{i M}+k_{p} W_{i}=0
$$

\subsubsection{Direct time integration method}

The direct time integration method developed here describes the dynamic response of the plate as multi-degree-offreedom system. The damping (viscosity $\eta)$ and an external loading $\mathrm{p}(\mathrm{x}, \mathrm{y}, \mathrm{t})$ are considered.

The governing equation applied at any point on the plate is as follows:

$$
\begin{aligned}
\frac{\partial^{4} w^{*}(x, y, t)}{\partial x^{4}}+2 \frac{\partial^{4} w^{*}(x, y, t)}{\partial x^{2} \partial y^{2}}+ & \frac{\partial^{4} w^{*}(x, y, t)}{\partial y^{4}}= \\
& \frac{1}{D}\left(p(x, y, t)-\rho d \frac{\partial^{2} w^{*}(x, y, t)}{\partial t^{2}}-\eta \frac{\partial w^{*}(x, y, t)}{\partial t}\right)
\end{aligned}
$$

The derivatives with respect to $\mathrm{x}$ and $\mathrm{y}$ are formulated similarly to previous sections; those with respect to $\mathrm{t}$ (the time increment is $\Delta \mathbf{t}$ ) are formulated considering a three-point stencil using Equations (45a-c),

$$
\left.\frac{\partial w^{*}(x, y, t)}{\partial t}\right|_{i, t}=\left.\frac{-w_{i, t-\Delta t}^{*}+w_{i, t+\Delta t}^{*}}{2 \Delta t} \quad \frac{\partial^{2} w^{*}(x, y, t)}{\partial t^{2}}\right|_{i, t}=\frac{w_{i, t-\Delta t}^{*}-2 w_{i, t}^{*}+w_{i, t+\Delta t}^{*}}{\Delta t^{2}}
$$


At the initial time $\mathbf{t}=\mathbf{0}$, a three-point forward difference approximation is applied

$$
\left.\frac{\partial^{2} w^{*}}{\partial t^{2}}\right|_{i, 0}=\left.\frac{w_{i, 0}^{*}-2 w_{i, \Delta t}^{*}+w_{i, 2 \Delta t}^{*}}{\Delta t^{2}} \quad \frac{\partial w^{*}}{\partial t}\right|_{i, 0}=\frac{-3 w_{i, 0}^{*}+4 w_{i, \Delta t}^{*}-w_{i, 2 \Delta t}^{*}}{2 \Delta t}
$$

At the final time $\mathbf{t}=\mathbf{T}$, a three-point backward difference approximation is applied

$$
\left.\frac{\partial^{2} w^{*}}{\partial t^{2}}\right|_{i, T}=\left.\frac{w_{i, T-2 \Delta t}^{*}-2 w_{i, T-\Delta t}^{*}+w_{i, T}^{*}}{\Delta t^{2}} \quad \frac{\partial w^{*}}{\partial t}\right|_{i, T}=\frac{w_{i, T-2 \Delta t}^{*}-4 w_{i, T-\Delta t}^{*}+3 w_{i, T}^{*}}{2 \Delta t}
$$

The governing equation (Equation (44)) can be formulated with FDM at any point of the plate at any time t. As described earlier, additional points are introduced to satisfy the boundary and continuity conditions. Thus, the plate deflection $\mathrm{w}^{*}(\mathrm{x}, \mathrm{y}, \mathrm{t})$ can be determined, and the efforts be calculated with appropriate formulas developed earlier.

\subsection{Analysis of isotropic Kirchhoff-Love plates of variable stiffness}

The flexural rigidity $\mathrm{D}$ of the plate may vary throughout the plate, and is then denoted $\mathrm{D}(\mathrm{x}, \mathrm{y})$. Substituting $\mathrm{D}(\mathrm{x}, \mathrm{y})$ into Equations (1a-c) yields the bending and twisting moments as follows

$$
\begin{aligned}
& m_{x x}=-D(x, y) \times\left(\frac{\partial^{2} w}{\partial x^{2}}+v \frac{\partial^{2} w}{\partial y^{2}}\right), \quad m_{y y}=-D(x, y) \times\left(\frac{\partial^{2} w}{\partial y^{2}}+v \frac{\partial^{2} w}{\partial x^{2}}\right), \\
& m_{x y}=-D(x, y) \times(1-v) \times \frac{\partial^{2} w}{\partial x \partial y}
\end{aligned}
$$

The substitution of Equation (46) into (3) yields the following governing equation

$$
\begin{aligned}
\frac{\partial^{2}}{\partial x^{2}}\left[D(x, y)\left(\frac{\partial^{2} w}{\partial x^{2}}+v \frac{\partial^{2} w}{\partial y^{2}}\right)\right]+ & 2 \frac{\partial^{2}}{\partial x \partial y}\left[D(x, y)(1-v) \frac{\partial^{2} w}{\partial x \partial y}\right]+ \\
& \frac{\partial^{2}}{\partial y^{2}}\left[D(x, y)\left(\frac{\partial^{2} w}{\partial y^{2}}+v \frac{\partial^{2} w}{\partial x^{2}}\right)\right]=q(x, y)
\end{aligned}
$$

The Kirchhoff shear forces are expressed using Equations (46) as follows

$$
V_{x}=\frac{\partial m_{x x}}{\partial x}+2 \frac{\partial m_{x y}}{\partial y}, \quad V_{y}=\frac{\partial m_{y y}}{\partial y}+2 \frac{\partial m_{x y}}{\partial x} .
$$

The FDAs of Equations (46) to (48) are formulated as described in previous sections, and the analysis continues similarly to that of the plate with constant stiffness. 


\section{$3 \quad$ Results and discussions}

\subsection{First-order analysis}

\subsubsection{Rectangular plate simply supported along all edges and subjected to a uniform load}

A rectangular plate simply supported along all edges and subjected to a uniformly distributed load was analyzed. The plate dimensions in $\mathrm{x}$ - and $\mathrm{y}$-directions are denoted by a and b, respectively, and the uniform load by q.

The bending moments at the middle of the plate, depending on the ratio b/a, are $m_{x x, m}=\mathrm{qa}^{2} / \mathrm{N}_{\mathrm{x}}$, and $\mathrm{m}_{\mathrm{yy}, \mathrm{m}}=\mathrm{qa}^{2} / \mathrm{N}_{\mathrm{y}}$. Details of the analysis and results are presented in Appendix A and in the supplementary material "Rectangular plate simply supported and subjected to a uniform load.” Table 1 lists the results obtained with Timoshenko [11] using the solution by Lévy [5] (exact results) and those obtained in the present study.

Table 1 Coefficients of bending moments at the middle of the plate

\begin{tabular}{|c|c|c|c|c|c|c|c|c|c|c|}
\hline \multirow[t]{2}{*}{$\lambda=\mathbf{b} / \mathbf{a}$} & \multicolumn{2}{|c|}{1.00} & \multicolumn{2}{|c|}{1.25} & \multicolumn{2}{|c|}{1.50} & \multicolumn{2}{|c|}{1.75} & \multicolumn{2}{|c|}{2.00} \\
\hline & $\mathbf{N}_{\mathbf{x}}$ & $\mathbf{N}_{\mathbf{y}}$ & $\mathbf{N}_{\mathbf{x}}$ & $\mathbf{N}_{\mathbf{y}}$ & $\mathbf{N}_{\mathbf{x}}$ & $\mathbf{N}_{\mathbf{y}}$ & $\mathbf{N}_{\mathbf{x}}$ & $\mathbf{N}_{\mathbf{y}}$ & $\mathbf{N}_{\mathbf{x}}$ & $\mathbf{N}_{\mathbf{y}}$ \\
\hline & \multicolumn{10}{|c|}{ Solution by Lévy [5] (exact results) } \\
\hline & 27.15 & 27.15 & 17.84 & 29.90 & 13.74 & 35.69 & 11.61 & 44.63 & 10.37 & 57.43 \\
\hline & \multicolumn{10}{|c|}{ FDM 25-pt Stencil } \\
\hline $8 \times 8$ elements & 28.00 & 28.00 & 18.39 & 30.73 & 14.15 & 36.44 & 11.92 & 45.09 & 10.62 & 57.23 \\
\hline $12 \times 12$ elements & 27.56 & 27.56 & 18.11 & 30.30 & 13.95 & 36.03 & 11.76 & 44.80 & 10.49 & 57.20 \\
\hline \multirow[t]{2}{*}{$16 \times 16$ elements } & 27.39 & 27.39 & 18.00 & 30.13 & 13.86 & 35.88 & 11.70 & 44.71 & 10.44 & 57.27 \\
\hline & \multicolumn{10}{|c|}{ FDM 13-pt Stencil } \\
\hline $8 \times 8$ elements & 27.48 & 27.48 & 18.08 & 30.17 & 13.94 & 35.83 & 11.78 & 44.45 & 10.52 & 56.56 \\
\hline $12 \times 12$ elements & 27.30 & 27.30 & 17.95 & 30.02 & 13.83 & 35.76 & 11.68 & 44.55 & 10.43 & 57.03 \\
\hline $16 \times 16$ elements & 27.23 & 27.23 & 17.90 & 29.97 & 13.79 & 35.73 & 11.65 & 44.58 & 10.40 & 57.20 \\
\hline
\end{tabular}

As Table 1 shows, the results of both approximations show good agreement with the exact results. Surprisingly, the 13pt stencil delivers better results than 25-pt stencil for a given grid; this is unexpected since the 25-pt stencil, contrarily to the 13-pt stencil, is consistent in the hypothesis of the deflection surface. A new 25-pt stencil should be investigated in future research, whereby a fourth-order polynomial hypothesis for the deflection surface would be considered at plate angles. 


\subsubsection{Rectangular SSFF plate subjected to a uniformly distributed load}

A rectangular plate simply supported along edges $\mathrm{x}=0$ and $\mathrm{x}=\mathrm{a}$, fixed along edges $\mathrm{y}=0$ and $\mathrm{y}=\mathrm{b}$, and subjected to a uniformly distributed load was analyzed. The bending moments, depending on the ratio $a / b$, are $m_{\text {yerm }}=-q^{2} / N_{\text {yerm, }}$, $\mathrm{m}_{\mathrm{xm}}=\mathrm{qb}^{2} / \mathrm{N}_{\mathrm{xm}}$, and $\mathrm{m}_{\mathrm{ym}}=\mathrm{qb}^{2} / \mathrm{N}_{\mathrm{ym}} ; \mathrm{m}_{\mathrm{yerm}}$ is the moment at the middle of the fixed edge, $\mathrm{m}_{\mathrm{xm}}$ and $\mathrm{m}_{\mathrm{ym}}$ are the bending moments at the middle of the plate in $\mathrm{x}$ - and $\mathrm{y}$-directions, respectively. Analysis and results are detailed in Appendix B and in the supplementary material "Rectangular SSFF plate subjected to a uniformly distributed load.” Table 2 displays the results obtained with Courbon [13] using the solution of Lévy [5] (exact results) and those obtained in the present study.

Table 2 Coefficients of bending moments in the plate

\begin{tabular}{|c|c|c|c|c|c|c|c|c|c|c|c|c|}
\hline \multirow[t]{3}{*}{$\lambda=\mathbf{a} / \mathbf{b}$} & \multicolumn{3}{|c|}{1.00} & \multicolumn{3}{|c|}{1.25} & \multicolumn{3}{|c|}{1.50} & \multicolumn{3}{|c|}{5.00} \\
\hline & \multicolumn{12}{|c|}{ Solution by Lévy (exact results) } \\
\hline & $\mathbf{N}_{\text {yerm }}$ & $\mathbf{N}_{\mathrm{xm}}$ & $\mathbf{N}_{\mathbf{y m}}$ & $\mathbf{N}_{\text {yerm }}$ & $\mathbf{N}_{\mathbf{x m}}$ & $\mathbf{N}_{\mathbf{y m}}$ & $\mathbf{N}_{\text {yerm }}$ & $\mathbf{N}_{\mathbf{x m}}$ & $\mathbf{N}_{\mathbf{y m}}$ & $\mathbf{N}_{\text {yerm }}$ & $\mathbf{N}_{\mathbf{x m}}$ & $\mathbf{N}_{\mathrm{ym}}$ \\
\hline & 14.32 & 63.13 & 35.10 & 12.78 & 96.80 & 28.50 & 12.17 & 162.15 & 25.79 & 12.00 & $\begin{array}{c}-410 \\
147.06\end{array}$ & 24.00 \\
\hline
\end{tabular}

FDM 25-pt Stencil

\begin{tabular}{|c|c|c|c|c|c|c|c|c|c|c|c|c|}
\hline & $\mathbf{N}_{\text {yerm }}$ & $\mathbf{N}_{\mathrm{xm}}$ & $\mathbf{N}_{\mathbf{y m}}$ & $\mathbf{N}_{\text {yerm }}$ & $\mathbf{N}_{\mathbf{x m}}$ & $\mathbf{N}_{\mathbf{y m}}$ & $\mathbf{N}_{\text {yerm }}$ & $\mathbf{N}_{\mathbf{x m}}$ & $\mathbf{N}_{\mathbf{y m}}$ & $\mathbf{N}_{\text {yerm }}$ & $\mathbf{N}_{\mathbf{x m}}$ & $\mathbf{N}_{\mathrm{ym}}$ \\
\hline $8 \times 8$ & 12.25 & 69.17 & 36.92 & 13.40 & 97.64 & 29.97 & 12.60 & 151.11 & 26.99 & 11.99 & $\begin{array}{c}-55 \\
297.45\end{array}$ & 23.97 \\
\hline $12 \times 12$ & 14.91 & 64.54 & 36.00 & 13.02 & 97.34 & 29.14 & 12.33 & 157.75 & 26.28 & 12.00 & $\begin{array}{c}-83 \\
360.31\end{array}$ & 23.99 \\
\hline $16 \times 16$ & 14.08 & 63.93 & 35.60 & 12.91 & 97.12 & 28.86 & 12.26 & 159.78 & 26.06 & 12.00 & $\begin{array}{c}-127 \\
152.51\end{array}$ & 24.00 \\
\hline
\end{tabular}

FDM 13-pt Stencil

\begin{tabular}{|c|c|c|c|c|c|c|c|c|c|c|c|c|}
\hline & $\mathbf{N}_{\text {yerm }}$ & $\mathbf{N}_{\mathrm{xm}}$ & $\mathbf{N}_{\mathbf{y m}}$ & $\mathbf{N}_{\text {yerm }}$ & $\mathbf{N}_{\mathrm{xm}}$ & $\mathbf{N}_{\mathbf{y m}}$ & $\mathbf{N}_{\text {yerm }}$ & $\mathbf{N}_{\mathbf{x m}}$ & $\mathbf{N}_{\mathbf{y m}}$ & $\mathbf{N}_{\text {yerm }}$ & $\mathbf{N}_{\mathrm{xm}}$ & $\mathbf{N}_{\mathbf{y m}}$ \\
\hline $8 \times 8$ & 15.40 & 57.59 & 35.60 & 13.43 & 85.49 & 28.55 & 12.63 & 136.33 & 25.63 & 12.19 & $\begin{array}{c}-359 \\
410.49\end{array}$ & 23.27 \\
\hline $12 \times 12$ & 14.77 & 60.43 & 35.32 & 13.05 & 91.33 & 28.52 & 12.36 & 149.58 & 25.71 & 12.08 & $\begin{array}{c}-154 \\
028.65\end{array}$ & 23.67 \\
\hline $16 \times 16$ & 14.57 & 61.56 & 35.22 & 12.93 & 93.63 & 28.51 & 12.27 & 154.85 & 25.74 & 12.05 & $\begin{array}{c}-183 \\
778.09\end{array}$ & 23.81 \\
\hline
\end{tabular}

The results of the present study show good agreement with the exact results, and the accuracy is increased through a grid refinement. 


\subsubsection{Rectangular plate simply supported along all edges and subjected to a non-uniform heating}

A rectangular plate simply supported along all edges and subjected to a non-uniform heating was analyzed. The plate dimensions in $\mathrm{x}$ - and $\mathrm{y}$-directions were denoted by a and b, respectively. The bending moments at the middle of the plate, depending on the ratio a/b and Poisson's ratio $v=0$, are $m_{x x, m}=D \kappa_{T} \times N_{x}$, and $m_{y y, m}=D \kappa_{T} \times N_{y}$. Detailed analysis and results are presented in Appendix C and in the Supplementary Material "Rectangular plate simply supported along all edges and subjected to a non-uniform heating.” Table 3 lists the results obtained by Fogang [14] using the solution by Lévy [5] and the Fourier sine transform method, and those obtained in the present study.

Table 3 Coefficients of bending moments at the middle of the plate

\begin{tabular}{|c|c|c|c|c|c|c|c|c|c|c|}
\hline \multirow[t]{2}{*}{$\lambda=\mathbf{a} / \mathbf{b}$} & \multicolumn{2}{|c|}{1.00} & \multicolumn{2}{|c|}{1.50} & \multicolumn{2}{|c|}{2.00} & \multicolumn{2}{|c|}{5.00} & \multicolumn{2}{|c|}{10.00} \\
\hline & $\mathbf{N}_{\mathbf{x}}$ & $\mathbf{N}_{\mathbf{y}}$ & $\mathbf{N}_{\mathbf{x}}$ & $\mathbf{N}_{\mathbf{y}}$ & $\mathbf{N}_{\mathbf{x}}$ & $\mathbf{N}_{\mathbf{y}}$ & $\mathbf{N}_{\mathbf{x}}$ & $\mathbf{N}_{\mathbf{y}}$ & $\mathbf{N}_{\mathbf{x}}$ & $\mathbf{N}_{\mathbf{y}}$ \\
\hline & \multicolumn{10}{|c|}{ Solution by Fogang [14] } \\
\hline & -0.500 & -0.506 & -0.762 & -0.245 & -0.890 & -0.116 & -0.999 & -0.007 & -1.000 & -0.006 \\
\hline & \multicolumn{10}{|c|}{ FDM 25-pt Stencil } \\
\hline $8 \times 8$ elements & -0.511 & -0.511 & -0.758 & -0.257 & -0.882 & -0.125 & -0.999 & 0.001 & -1.000 & 0.000 \\
\hline \multirow[t]{2}{*}{$12 \times 12$ elements } & -0.507 & -0.509 & -0.759 & -0.255 & -0.883 & -0.125 & -0.998 & -0.002 & -1.000 & 0.000 \\
\hline & \multicolumn{10}{|c|}{ FDM 13-pt Stencil } \\
\hline $8 \times 8$ elements & -0.500 & -0.500 & -0.754 & -0.246 & -0.881 & -0.119 & -0.997 & -0.003 & -1.000 & 0.000 \\
\hline $12 \times 12$ elements & -0.500 & -0.500 & -0.758 & -0.242 & -0.886 & -0.114 & -0.998 & -0.002 & -1.000 & 0.000 \\
\hline
\end{tabular}

The results of the present study show good agreement with the exact results, and the accuracy is increased through a grid refinement. 


\subsection{Second-order analysis}

\subsubsection{Rectangular plate simply supported along all edges and subjected to a uniformly distributed load and a compressive force}

A rectangular plate simply supported along all edges and subjected to a uniformly distributed load $\mathrm{q}$ and compressive forces $N_{x}$ was analyzed. The compressive forces were applied along edges $\mathrm{x}=0$ and $\mathrm{x}=\mathrm{a}$, whereby a and $\mathrm{b}$ were the plate dimensions in $x$ - and $y$-directions, respectively. The axial force $N_{x}$ is such that $N_{x} a^{2} / \pi^{2} / D=-1.0$. The bending moments at the middle of the plate, depending on the ratio b/a, are $\mathrm{m}_{\mathrm{xx}, \mathrm{m}}=\mathrm{qa}^{2} / \mathrm{C}_{\mathrm{x}}$, and $\mathrm{m}_{\mathrm{yy}, \mathrm{m}}=\mathrm{qa}^{2} / \mathrm{C}_{\mathrm{y}}$. Details of the analysis and results are presented in Appendix D and in the supplementary material "Rectangular plate simply supported and subjected to a uniform load and a compressive force.” Table 4 lists the results obtained with Timoshenko [11] using the solution by Navier [4] (exact results) and those obtained in the present study.

Table 4 Coefficients of bending moments at the middle of the plate

\begin{tabular}{|c|c|c|c|c|c|c|c|c|c|c|}
\hline \multirow[t]{3}{*}{$\lambda=\mathbf{b} / \mathbf{a}$} & \multicolumn{2}{|c|}{1.00} & \multicolumn{2}{|c|}{1.25} & \multicolumn{2}{|c|}{1.50} & \multicolumn{2}{|c|}{2.00} & \multicolumn{2}{|c|}{5.00} \\
\hline & $C_{x}$ & $\mathbf{C}_{\mathbf{y}}$ & $\mathbf{C}_{\mathbf{x}}$ & $\mathbf{C}_{\mathbf{y}}$ & $\mathbf{C}_{\mathbf{x}}$ & $\mathbf{C}_{\mathbf{y}}$ & $\mathbf{C}_{\mathbf{x}}$ & $\mathbf{C}_{\mathbf{y}}$ & $\mathbf{C}_{\mathbf{x}}$ & $\mathbf{C}_{\mathbf{y}}$ \\
\hline & \multicolumn{10}{|c|}{ Solution by Navier (exact results) } \\
\hline & 19.97 & 19.83 & 10.91 & 17.73 & 6.92 & 16.71 & 3.54 & 15.88 & 0.51 & 15.50 \\
\hline & \multicolumn{10}{|c|}{ FDM 25-pt Stencil } \\
\hline $8 \times 8$ elements & 20.52 & 20.35 & 11.16 & 18.08 & 7.02 & 16.87 & 3.52 & 15.59 & 0.36 & 10.33 \\
\hline $12 \times 12$ elements & 20.25 & 20.09 & 11.04 & 17.91 & 6.98 & 16.80 & 3.54 & 15.76 & 0.45 & 13.12 \\
\hline \multirow[t]{2}{*}{$16 \times 16$ elements } & 20.13 & 19.98 & 10.99 & 17.84 & 6.95 & 16.76 & 3.54 & 15.81 & 0.48 & 14.15 \\
\hline & \multicolumn{10}{|c|}{ FDM 13-pt Stencil } \\
\hline $8 \times 8$ elements & 20.13 & 19.99 & 10.97 & 17.78 & 6.93 & 16.64 & 3.51 & 15.56 & 0.43 & 12.63 \\
\hline $12 \times 12$ elements & 20.04 & 19.90 & 10.94 & 17.75 & 6.92 & 16.68 & 3.53 & 15.74 & 0.48 & 14.20 \\
\hline $16 \times 16$ elements & 20.01 & 19.87 & 10.92 & 17.74 & 6.92 & 16.69 & 3.53 & 15.80 & 0.49 & 14.77 \\
\hline
\end{tabular}

The results of the present study show good agreement with the exact results, and the accuracy is increased through a grid refinement. 


\subsubsection{Plate buckling of a rectangular plate simply supported along all edges and subjected to a compressive force}

The plate buckling analysis of a rectangular plate simply supported along all edges and subjected to compressive forces $\mathrm{N}_{\mathrm{x}}$ was conducted. The compressive forces were applied along edges $\mathrm{x}=0$ and $\mathrm{x}=\mathrm{a}$, whereby a and $\mathrm{b}$ were the plate dimensions in $\mathrm{x}$ - and y-directions, respectively. The critical axial force $\mathrm{N}_{\mathrm{x}, \mathrm{rr}}$ is defined as follows $\mathrm{N}_{\mathrm{x}, \mathrm{cr}}=-\mathrm{k} \pi^{2} \mathrm{D} / \mathrm{b}^{2}$; $\mathrm{k}$ is the plate buckling factor. Bryan [15] derived following expressions of the buckling load and buckling factor

$$
N_{x, c r}=-\frac{\pi^{2} D}{b^{2}}\left(\frac{m b}{a}+\frac{a}{m b}\right)^{2}, \quad k=\left(\frac{m b}{a}+\frac{a}{m b}\right)^{2}
$$

Details of the results are presented in the supplementary material "Plate buckling of a rectangular plate simply supported and subjected to a compressive force.” Table 5 displays the buckling factors, depending on the ratio a/b, obtained using Equation (49) by Bryan [15] and those obtained in the present study.

Table 5 Buckling factor $\mathrm{k}$ for the plate subjected to compressive forces along edges $\mathrm{x}=0$ and $\mathrm{x}=\mathrm{a}$

\begin{tabular}{|c|c|c|c|c|c|c|c|c|c|c|}
\hline$\lambda=\mathbf{a} / \mathbf{b}$ & 0.10 & 0.50 & 0.75 & 1.00 & $\sqrt{2}$ & 2.00 & $\sqrt{6}$ & 3.00 & $\sqrt{12}$ & 4.00 \\
\hline & \multicolumn{10}{|c|}{ Solution by Bryan [15] } \\
\hline & 102,01 & 6,250 & 4,340 & 4,000 & 4,500 & 4,000 & 4,167 & 4,000 & 4,083 & 4,000 \\
\hline & \multicolumn{10}{|c|}{ FDM 25-pt Stencil } \\
\hline $8 \times 8$ elements & 97.77 & 6.143 & 4.281 & 3.949 & 4.299 & 3.882 & 3.872 & 3.799 & 3.699 & 3.617 \\
\hline $12 \times 12$ elements & 100.88 & 6.202 & 4.314 & 3.977 & 4.407 & 3.945 & 4.020 & 3.858 & 3.847 & 3.838 \\
\hline \multirow[t]{2}{*}{$16 \times 16$ elements } & 100.82 & 6.223 & 4.325 & 3.987 & 4.446 & 3.969 & 4.081 & 3.938 & 3.955 & 3.903 \\
\hline & \multicolumn{10}{|c|}{ FDM 13-pt Stencil } \\
\hline $8 \times 8$ elements & 100.71 & 6.170 & 4.285 & 3.949 & 4.385 & 3.948 & 3.860 & 3.960 & 3.956 & 3.872 \\
\hline $12 \times 12$ elements & 101.43 & 6.214 & 4.316 & 3.977 & 4.449 & 3.977 & 4.107 & 3.979 & 4.018 & 3.985 \\
\hline $16 \times 16$ elements & 101.68 & 6.230 & 4.326 & 3.987 & 4.471 & 3.987 & 4.133 & 3.988 & 4.044 & 3.989 \\
\hline
\end{tabular}

The results of the present study show good agreement with the results of Bryan [15], and the accuracy is increased through a grid refinement. However, special attention should be taken when solving the eigenvalue problem, since confusion between the modes is easily done; an efficient tool may be needed for this purpose. 


\subsection{Vibration analysis}

\subsubsection{Free vibration analysis of a rectangular plate simply supported along all edges}

The free vibration analysis of a rectangular plate simply supported along all edges was conducted. The plate dimensions in $\mathrm{x}$ - and $\mathrm{y}$-directions were denoted by a and b, respectively. The vibration frequency $\omega$ is defined as follows:

$$
\omega=\lambda^{*} \times \sqrt{\frac{D}{\rho d a^{4}}}
$$

The circular frequency for this special case is widely known in the literature (see Wikipedia [16]); its general formulation and the first mode $\quad(m=n=1)$ are given as follows

$$
\omega_{m n}=\left(\frac{m^{2}}{a^{2}}+\frac{n^{2}}{b^{2}}\right) \sqrt{\frac{D \pi^{4}}{\rho d}} \quad \omega_{1,1}=\pi^{2}\left(1+\frac{a^{2}}{b^{2}}\right) \sqrt{\frac{D}{\rho d a^{4}}}
$$

Details of the results are presented in the supplementary material "Free vibration analysis of a rectangular plate simply supported along all edges.” Table 6 displays the free vibration factors $\lambda^{*}$ (Equation (50)), depending on the ratio a/b, obtained using Equation (51b) by Wikipedia [16] and those obtained in the present study.

\begin{tabular}{|c|c|c|c|c|c|c|c|c|c|c|}
\hline$\lambda=\mathbf{a} / \mathbf{b}$ & 0.10 & 0.50 & 0.75 & 1.00 & 1.50 & 2.00 & 2.50 & 3.00 & 3.50 & 4.00 \\
\hline & \multicolumn{10}{|c|}{ Solution by Wikipedia [16] } \\
\hline & 9,968 & 12,337 & 15,421 & 19,739 & 32,076 & 49,348 & 71,555 & 98,696 & 130,77 & 167,78 \\
\hline & \multicolumn{10}{|c|}{ FDM 25-pt Stencil } \\
\hline $8 \times 8$ elements & 9.842 & 12.229 & 15.313 & 19.611 & 31.838 & 48.916 & 70.854 & 97.658 & 129.33 & 165.87 \\
\hline \multirow[t]{2}{*}{$12 \times 12$ elements } & 9.913 & 12.289 & 15.374 & 19.683 & 31.971 & 49.156 & 71.243 & 98.234 & 130.13 & 166.93 \\
\hline & \multicolumn{10}{|c|}{ FDM 13-pt Stencil } \\
\hline $8 \times 8$ elements & 9.841 & 12.179 & 15.224 & 19.487 & 31.666 & 48.717 & 70.640 & 97.434 & 129.11 & 165.64 \\
\hline $12 \times 12$ elements & 9.911 & 12.267 & 15.333 & 19.627 & 31.893 & 49.067 & 71.147 & 98.13 & 130.09 & 166.83 \\
\hline
\end{tabular}

Table 6 Coefficients $\lambda^{*}$ of natural frequencies (first mode) of a rectangular plate

The results of the present study show good agreement with the exact results, and the accuracy is increased through a grid refinement. However, special attention should be taken when solving the eigenvalue problem, since confusion between the modes is easily done; an efficient tool may be needed for this purpose. 
KIRCHHOFF-LOVE PLATE THEORY USING THE FINITE DIFFERENCE METHOD

\section{Conclusions}

The FDM-based model developed in this paper enabled, with relative easiness, first-order analysis, second-order analysis, and vibration analysis of Kirchhoff-Love plates. The results showed that the calculations conducted as described in this paper were accurate. The 13-pt stencil and 25-pt stencil presented deliver good results, whereby the results of 13-pt stencil were better for a given grid. A new 25-pt stencil should be investigated in in future research, whereby a fourth-order polynomial hypothesis for the deflection surface would be considered at plate angles and at skew edges.

The following aspects were not addressed in this study but could be analyzed with the model in future research:

Consideration of Poisson’s ratio

Analysis of anisotropic plates

Plates resting on Pasternak foundations.

\section{However, some study limitations should be acknowledged}

Large deformation theory

Supplementary Materials: The following files were uploaded during submission:

- "Rectangular plate simply supported along all edges and subjected to a uniform load,"

- " $\quad$ Rectangular SSFF plate subjected to a uniformly distributed load,"

- "Rectangular plate simply supported along all edges and subjected to a non-uniform heating,”

- "Rectangular plate simply supported and subjected to uniform load and compressive force,"

- " $\quad$ Plate buckling of a rectangular plate simply supported and subjected to a compressive force,"

- "Free vibration analysis of a rectangular plate simply supported along all edges.”

Only the 8 x 8 element discretization was uploaded in these supplementary files.

\section{Author Contributions:}

\section{Funding:}

\section{Acknowledgments:}

Conflicts of Interest: The author declares no conflict of interest. 


\section{Appendix A Rectangular plate simply supported along all edges and subjected to a uniform load}

Timoshenko [11] presented following expressions for bending moments along the x-axis using the solution by Lévy [5].

$$
\begin{aligned}
& m_{x x, y=0}=\frac{q x(a-x)}{2}-q a^{2} \pi^{2} \times \sum_{m=1,3,5 \ldots} m^{2}\left[2 v B_{m}-(1-v) A_{m}\right] \sin \frac{m \pi x}{a} \\
& m_{y y, y=0}=v \frac{q x(a-x)}{2}-q a^{2} \pi^{2} \times \sum_{m=1,3,5 \ldots} m^{2}\left[2 B_{m}+(1-v) A_{m}\right] \sin \frac{m \pi x}{a} \\
& A_{m}=-\frac{2\left(\alpha_{m} \tanh \alpha_{m}+2\right)}{\pi^{5} m^{5} \cosh \alpha_{m}}, \quad B_{m}=\frac{2}{\pi^{5} m^{5} \cosh \alpha_{m}}, \quad \alpha_{m}=\frac{m \pi b}{2 a} .
\end{aligned}
$$

The bending moments at the middle of the plate $(x=a / 2, y=0)$ for Poisson's ratio $v=0$ are deduced as follows

$$
\begin{aligned}
& m_{x x, m}=\frac{q a^{2}}{8}+q a^{2} \pi^{2} \times \sum_{m=1,3,5 \ldots} m^{2} A_{m} \sin \frac{m \pi}{2} \\
& m_{y y, m}=-q a^{2} \pi^{2} \times \sum_{m=1,3,5 \ldots} m^{2}\left(A_{m}+2 B_{m}\right) \sin \frac{m \pi}{2}
\end{aligned}
$$

\section{Appendix B Rectangular SSFF plate subjected to a uniformly distributed load}

The plate is simply supported along edges $\mathrm{x}=0$ and $\mathrm{x}=\mathrm{a}$, fixed along edges $\mathrm{y}=0$ and $\mathrm{y}=\mathrm{b}$, and subjected to a uniformly distributed load. Courbon [13] derived following expressions for bending moments, namely those at the middle of the fixed edges and at the middle of the plate, using the solution by Lévy [5]; $\mathrm{m}_{\text {yerm }}$ is the moment at the middle of the fixed edges, $\mathrm{m}_{\mathrm{xm}}$ and $\mathrm{m}_{\mathrm{ym}}$ are the bending moments at the middle of the plate in $\mathrm{x}$ - and $\mathrm{y}$-directions, respectively.

$$
\begin{aligned}
& m_{\text {yerm }}=-\frac{1}{12} p b^{2} H_{1}(\rho) \\
& m_{x, m}=\frac{1}{24} p b^{2}\left[F_{1}(\rho)+v G_{1}(\rho)\right], \quad m_{y, m}=\frac{1}{24} p b^{2}\left[v F_{1}(\rho)+G_{1}(\rho)\right] \\
& F_{1}(\rho)=3 \rho^{2} F(\rho), \quad G_{1}(\rho)=3 \rho^{2} G(\rho), \quad H_{1}(\rho)=\frac{3}{2} \rho^{2} H(\rho) \\
& F(\rho)=1-\sum_{m=1,3,5 . .} \frac{32}{m^{3} \pi^{3}}\left(\frac{\lambda \times \operatorname{ch} \lambda+\operatorname{sh} \lambda}{\operatorname{sh} \lambda \times \operatorname{ch} \lambda+\lambda}\right) \sin \frac{m \pi}{2}, \quad G(\rho)=\sum_{m=1,3,5 \ldots} \frac{32}{m^{3} \pi^{3}}\left(\frac{\lambda \times \operatorname{ch} \lambda-\operatorname{sh} \lambda}{\operatorname{sh} \lambda \times \operatorname{ch} \lambda+\lambda}\right) \sin \frac{m \pi}{2} \\
& H(\rho)=1-\sum_{m=1,3,5 \ldots} \frac{64}{m^{3} \pi^{3}}\left(\frac{2 \lambda}{\operatorname{sh} 2 \lambda+2 \lambda}\right) \sin \frac{m \pi}{2} \\
& \rho=\frac{a}{b} \quad \lambda=\frac{m \pi b}{2 a} \text {. }
\end{aligned}
$$




\section{Appendix C Rectangular plate simply supported along all edges and subjected to a non-uniform heating}

The governing equation of the isotropic plate subjected to a non-uniform heating is given by

$$
\frac{\partial^{4} w}{\partial x^{4}}+2 \frac{\partial^{4} w}{\partial x^{2} \partial y^{2}}+\frac{\partial^{4} w}{\partial y^{4}}=-\frac{\partial^{2}(1+v) \kappa_{T}}{\partial x^{2}}-\frac{\partial^{2}(1+v) \kappa_{T}}{\partial y^{2}}
$$

The right-hand side of Equation (C1) is zero for a non-uniform heating constant throughout the plate. The bending moments per unit length $\mathrm{m}_{\mathrm{xx}}$ and $\mathrm{m}_{\mathrm{yy}}$ are given by

$$
\begin{aligned}
& m_{x x}=-D \times\left(\frac{\partial^{2} w}{\partial x^{2}}+v \frac{\partial^{2} w}{\partial y^{2}}+(1+v) \kappa_{T}\right), \\
& m_{y y}=-D \times\left(\frac{\partial^{2} w}{\partial y^{2}}+v \frac{\partial^{2} w}{\partial x^{2}}+(1+v) \kappa_{T}\right) .
\end{aligned}
$$

\section{Appendix D Rectangular plate simply supported along all edges and subjected to a uniformly distributed load and a compressive force}

Timoshenko [11] derived following expression for the deflection surface using the solution by Navier [4].

$$
w(x, y)=\frac{16 q}{\pi^{6} D} \times \sum_{m=1,3,5 \ldots} \sum_{n=1,3,5 \ldots} \frac{1}{m n\left[\left(\frac{m^{2}}{a^{2}}+\frac{n^{2}}{b^{2}}\right)^{2}+\frac{N_{x} m^{2}}{\pi^{2} D a^{2}}\right]} \sin \frac{m \pi x}{a} \sin \frac{n \pi y}{b}
$$

The bending moments $\mathrm{m}_{\mathrm{xx}}(\mathrm{x}, \mathrm{y})$ and $\mathrm{m}_{\mathrm{yy}}(\mathrm{x}, \mathrm{y})$ in $\mathrm{x}$ - and $\mathrm{y}$-directions, respectively, are obtained using Equations (1a-b) with the Poisson's ratio $v=0$

$m_{x x}(x, y)=\frac{16 q a^{2}}{\pi^{4}} \times \sum_{m} \sum_{n} \frac{m}{n} \frac{1}{\left[\left(m^{2}+(n a / b)^{2}\right)^{2}+\frac{N_{x} a^{2} m^{2}}{\pi^{2} D}\right]} \sin \frac{m \pi x}{a} \sin \frac{n \pi y}{b}$

$m_{x x}(x, y)=\frac{16 q a^{2}}{\pi^{4}} \times \frac{a^{2}}{b^{2}} \sum_{m} \sum_{n} \frac{n}{m} \frac{1}{\left[\left(m^{2}+(n a / b)^{2}\right)^{2}+\frac{N_{x} a^{2} m^{2}}{\pi^{2} D}\right]} \sin \frac{m \pi x}{a} \sin \frac{n \pi y}{b}$ 
KIRCHHOFF-LOVE PLATE THEORY USING THE FINITE DIFFERENCE METHOD

\section{References}

[1] Fogang, V. Euler-Bernoulli Beam Theory: First-Order Analysis, Second-Order Analysis, Stability, and Vibration Analysis Using the Finite Difference Method. Preprints 2021, 2021020559

(doi: 10.20944/preprints202102.0559.v3).

[2] Kirchhoff, G. Über das Gleichgewicht und die Bewegung einer elastischen Scheibe. J. für die Reine und Angew. Math.; vol. 18, no. 40, pp. 51-88, 1850.

[3] Germain, S. Remarques sur la nature, les bornes et l'étendue de la question des surfaces élastiques et équation générale de ces surfaces. impr. de Huzard-Courcier, paris, 1826.

[4] Navier, C.L. Extrait des recherches sur la flexion des plaques élastiques. Bull. des Sci. Société Philomath, Paris, vol. 10, no. 1, pp. 92-102, 1823.

[5] Lévy, M. Sur l'équilibre élastique d'une plaque rectangulaire. Comptes rendus l’Académie des Sci. Paris, vol. 129, no. 1, pp. 535-539, 1899.

[6] Kindelan, M., Moscoso, M., Gonzalez-Rodriguez, P. Optimized Finite Difference Formulas for Accurate High Frequency Components. Mathematical Problems in Engineering. Volume 2016, Article ID 7860618, 15 pages. http://dx.doi.org/10.1155/2016/7860618

[7] Onyia, M.E., Rowland-Lato, E.O., Ike, C.C. Elastic buckling analysis of SSCF and SSSS rectangular thin plates using the single finite Fourier sine integral transform method. IJERT, Vol 13, No 6, pp 1147 - 1158, july 2020. DOI: 10.37624/IJERT/13.6.2020.1147-1158

[8] Pisacic, K., Horvat, M., Botak, Z. Finite Difference Solution of Plate Bending Using Wolfram Mathematica. Tehnicki Glasnik 13, 3(2019), 241-247. https://doi.org/10.31803/tg-20190328111708

[9] Li, Y-D., Tang, Z-C., Fu, Z-J. Generalized Finite Difference Method for Plate Bending Analysis of Functionally Graded Materials. Mathematics 2020, 8, 1940; http://dx.doi.org/10.3390/math8111940

[10] Ferreira, A.C.A., Ribeiro, P.M.V. Reduced-order strategy for meshless solution of plate bending problems with the generalized finite difference method. Lat. Am. j. solids struct. 16 (01). 2019. https://doi.org/10.1590/1679-78255191

[11] Timoshenko, S., Woinowsky-Krieger, S. Theory of plates and shells. McGraw-Hill Book Company, $2^{\text {nd }}$ edition, 1959. ISBN 0-07-085820-9

[12] Clebsch, A., Saint Venant, B., Flamant, A.A . Théorie de l'élasticité des corps solides. Paris, Dunod, 1883 (final note 73), p. 704.

[13] Courbon, J., Theillout, JN. Formulaire de résistance des matériaux. Techniques de l’Ingénieur, traité Construction. 1987. https://www.techniques-ingenieur.fr/base-documentaire/archives-th12/archives-le-secondoeuvre-et-I-equipement-du-batiment-tiacc/archive-1/resistance-des-materiaux-c2060/ 
[14] Fogang, V. Bending Analysis of Isotropic Rectangular Kirchhoff Plates Subjected to a Thermal Gradient Using the Fourier Transform Method . Preprints 2021, 2021060479 (doi: 10.20944/preprints202106.0479.v1).

[15] Bryan, C.H. On the stability of a plane plate under thrusts in its own plane, with application in the buckling of the sides of a ship. London Math. Society Proc.22, 1891, p. 54-67.

[16] Wikipedia ...https://en.wikipedia.org/wiki/Vibration_of_plates 